\title{
$1 \quad$ PYK2 controls intestinal inflammation via activation of IRF5 in macrophages
}

2 Grigory Ryzhakov ${ }^{1 \dagger *}$, Hannah Almuttaqi ${ }^{1 *}$, Alastair L. Corbin ${ }^{1}$, Tariq Khoyratty ${ }^{1}$, Dorothee

3 Berthold $^{1}$, Samuel Bullers ${ }^{1}$, Hayley L Eames ${ }^{1}$, Zhichao Ai ${ }^{1}$, Sarah Bonham ${ }^{3}$, Roman Fischer ${ }^{3}$,

4 Luke Jostins-Dean ${ }^{1}$, Simon P.L. Travis ${ }^{2}$, Benedikt M. Kessler ${ }^{3}$ and Irina A. Udalova ${ }^{1 \#}$

5

$6 \quad{ }^{1}$ University of Oxford, Kennedy Institute of Rheumatology, Oxford, United Kingdom

$7 \quad{ }^{2}$ Translational Gastroenterology Unit, NIHR Oxford Biomedical Research Centre, Oxford

8 University Hospitals NHS Foundation Trust, John Radcliffe Hospital, Oxford, United Kingdom

$9{ }^{3}$ Target Discovery Institute, Nuffield Department of Medicine, University of Oxford, Oxford,

10 United Kingdom

11 present address - Novartis Institutes for BioMedical Research, Novartis Pharma AG, Novartis

12 Campus, Basel, Switzerland

13

$14 \quad$ "correspondent author - irina.udalova@kennedy.ox.ac.uk

$15 *$ These authors contributed equally to this work. 


\section{Abstract}

18 Inflammatory bowel disease (IBD) is a group of inflammatory disorders of the gastro-intestinal

19 tract caused by a complex combination of genetic and environmental factors. Interferon

20 regulating factor 5 (IRF5) is a multifunctional regulator of immune responses, which plays a

21 key pathogenic role in mouse colitis models and is a genetic risk factor for IBD. A screen of a protein kinase inhibitor library in macrophages revealed a list of putative IRF5 kinases. Among the top hits validated in multiple in vitro assays, protein-tyrosine kinase 2-beta (PTK2B or PYK2) was identified as the only IBD genetic risk factor, known to impact gene expression in myeloid cells ${ }^{1,2}$. Phospho-proteomics and mutagenesis analyses established that PYK2 directly 26 phosphorylates and activates IRF5 at tyrosine (Y) 171. IRF5 nuclear translocation and recruitment to target genes was impaired in PYK2-deficient cells or in cells treated with PYK2 inhibitors. Importantly, macrophage transcriptomic signature under PYK2 inhibition phenocopied IRF5 deficiency. Treatment with a PYK2 inhibitor reduced pathology and

30 inflammatory cytokine production in Helicobacter hepaticus + anti-IL-10R antibody induced

31 colitis model. It also decreased levels of pro-inflammatory cytokines in human colon biopsies

32 taken from patients with ulcerative colitis. Thus, we have identified a major role for PYK2 in regulating the inflammatory response and mapped its activity to the IRF5 innate sensing

34 pathway, opening opportunities for therapeutic interference with it in IBD and other 35 inflammatory conditions. 
37 A recent single-cell transcriptomic analysis of colon biopsies from patients with ulcerative colitis (UC) provided a framework for linking GWAS risk loci with specific cell types and functional pathways and helped to nominate causal genes across GWAS loci ${ }^{3}$, amongst them

40 Interferon regulatory factor 5 (IRF5). IRF5 is a multifunctional regulator of immune responses $^{4-6}$. The IRF5 risk variant has consistent effects across monocytes and macrophage conditions, but also impacts gene expression and splicing across a wide range of other immune cells and tissues ${ }^{7}$.

Recent studies using IRF5-deficient mice have established a critical role of this transcription factor in the pathogenesis of mouse models of colitis ${ }^{8,9}$. IRF5 is proposed to exert its molecular function via a cascade of events involving its phosphorylation, ubiquitination, dimerisation, nuclear translocation and selective binding to its target genes to enable their expression ${ }^{10}$. Despite its known physiological role, the molecular mechanisms of IRF5 activation are still debated. Several kinases including TBK1, RIP2, IKKe, IRAK4, TAK1, and IKK $\beta$ have been proposed to phosphorylate and activate IRF5 ${ }^{11-17}$, while IKK $\alpha$ inhibits IRF5 ${ }^{18}$. Lyn, a Src family kinase has been shown negatively to regulate IRF5 in the TLR-MyD88 pathway in a kinase independent manner via direct binding to IRF5 ${ }^{19}$.

In this study, we identified another nominated causal gene for $\mathrm{UC}^{3}$, Protein Tyrosine Kinase 2b (PTK2B/PYK2), as a key regulator of IRF5 activation, macrophage inflammatory response, and intestinal pathology extending its currently accepted function in macrophage morphology and migration ${ }^{20}$.

We have previously established an in vitro reporter system for measuring IRF5-dependent transcription based on the TNF (IRF5-dependent gene)-promoter driven luciferase construct, which contains a number of interferon-stimulated response elements (ISREs) ${ }^{21}$. In our hands 
the TNF promoter reporter consistently showed a stronger response to IRF5 than the standardly

62

used ISRE-luciferase reporter (Supplementary Fig. 1a, b), either due to the number of ISRE sites and/or previously reported IRF5 cooperation with NF-kB RelA ${ }^{22}$. This reporter system was used to screen a library of small molecules ${ }^{23}$, for which inhibitory properties against 221 protein kinases in the Protein Kinase Inhibitors Screen (PKIS) have been established (Supplementary Fig. 1c, d, e). After the first screen in RAW264.7 macrophages and 2 rounds of re-screening using different cell types and three different inhibitor concentrations, we composed the final list of 34 candidate IRF5 kinases, among which TBK1, IKKe and IRAK4 were previously proposed to target IRF5 $5^{12,16,24}$ (Fig. 1a, Source Data 1).

For further functional validation we selected poorly explored proteins or those with known links to inflammatory processes - PYK2, HIPK4, ARK5, CLK2, MARK3, JNK2 and MST1. We then cloned the cDNAs encoding them into mammalian expression vectors and tested them in functional assays. As controls, we included into these assays RIP2 kinase, the known intermediate in the IRF5-dependent innate immune signalling pathways ${ }^{11}$. When we overexpressed the kinases with IRF5 and TNF-luciferase reporter in HEK-293 TLR4/CD14/MD-2 cells, we found that overexpression of PYK2, JNK2 or MARK3, boosted IRF5-dependent TNF-reporter activation (Fig. 1b). Similar to the known IRF5 binding partners RIP2 and RelA ${ }^{11,22,25}$, PYK2 could strongly bind IRF5 in co-immunoprecipitation assays, while HIPK4, ARK5 and JNK2 showed only weak association with IRF5 (Fig. 1c). We further tested the ability of these kinases to phosphorylate overexpressed IRF5 in 293 ET cell lysates (Supplementary Fig. 1f). We were able to detect phosphorylated IRF5 in the presence of HIPK4, CLK2, JNK2, MST1, PYK2 and RIP2 as a positive control (Fig. 1d). Lastly, we examined the evidence of genetic association of the selected kinases with IBD and found that PYK2 was the only known genetic risk factor ${ }^{26}$. Moreover, the risk variant for PYK2 was 
shown to impact gene expression in monocytes and macrophages ${ }^{27}$. Based on observed functional interactions with IRF5 and genetic association with IBD, PYK2 was singled out for further investigation in macrophages (Supplementary Fig. 1g).

In RAW264.7 macrophages we could detect PYK2 binding to IRF5 at the endogenous level (Fig. 2a). In line with previous studies, we also found LPS-induced PYK2 phosphorylation on Y402 (Fig. 2b) ${ }^{28,29}$. To investigate the kinase's role in the TLR4/IRF5 signalling axis, we generated IRF5- and PYK2-deficient mouse RAW264.7 macrophages using a CRISPR-Cas9 approach (Supplementary Fig. 2a). First, we explored the impact of PYK2 deficiency on IRF5-dependent signalling by transfecting wild type (WT) and PYK2-deficient RAW264.7 macrophages with IRF5-expressing and the TNF-promoter driven luciferase plasmid. We observed a marked reduction of the LPS-induced reporter activity in IRF5 expressing cells lacking PYK2 (Fig. 2c). When we expressed recombinant PYK2 in PYK2-deficient cells, we achieved a partial reconstitution of the PYK2 levels in RAW264.7 macrophages and a partial restoration of the reporter activity (Supplementary Fig. 2b, c). Next, we examined if PYK2 deficiency would directly impact IRF5 activation and function by measuring IRF5 recruitment to its target gene promoter and enhancer regions using chromatin immunoprecipitation (ChIP) assay $^{4,21}$. IRF5 recruitment to Il6, Il la and Tnf gene promoters was impaired in PYK2 knockout cells (Fig. 2d, Supplementary Fig. 2d). Consequently, we observed attenuated recruitment of RNA polymerase II at the same promoters indicating reduced gene transcription (Fig. 2d, Supplementary Fig. 2d). We also detected reduction in mRNA induction of these cytokines, as well as chemokines $C c l 4, C c l 5$, by LPS in PYK2-deficient cells, comparable to or even stronger than in IRF5 knockout cells (Fig. 2e, Supplementary Fig. 2e). Conversely, LPSinduced IL-10 induction was increased in PYK2 knockout (Supplementary Fig. 2e), similarly to our previous findings in IRF5 knockout cells ${ }^{4}$. 
111 To validate our observations in primary cells, we utilised immortalised myeloid progenitor

112 HoxB8 cells ${ }^{30}$, which differentiated into non-proliferating mature macrophages after GM-CSF-

113 induced differentiation for 5 days (Supplementary Fig. 3a). Using the CRISP-Cas9 approach,

114 we generated stable knockout of IRF5 and PYK2 in these cells and validated their absence by

115 western blot analysis (Supplementary Fig. 3b). After 5 days of ex vivo differentiation in the

116 presence of GM-CSF, HoxB8 progenitors deficient in IRF5 or PYK2 gave rise to mature

117 macrophages, comparable to the wt cells, but the levels of inflammatory cytokine and

118 chemokine production were significantly reduced in HoxB8 macrophages deficient in IRF5 or

119 PYK2 (Supplementary Fig. 3c). Thus, PYK2 acts as a critical regulator of IRF5-dependent

120 transcription and inflammatory response induced by LPS in macrophages.

121

To characterise potential PYK2 target residues in IRF5, we employed phospho-proteomics.

Endogenous IRF5 was immuno-precipitated from the lysates of LPS-stimulated WT and PYK2-deficient RAW264.7 macrophages, and the phospho-peptides were further enriched from the total proteolytic digests. Peptide masses and quantities were analysed by nano ultrahigh-pressure liquid chromatography coupled with mass spectrometry (nUPLC-MS/MS). In

127 line with previously published reports ${ }^{14,15}$ we identified the Ser-445 IKK $\beta$-dependent site in both WT and PYK2-deficient cells (Fig. 2f, Supplementary Fig. 4a, b). In addition, endogenous IRF5 was phosphorylated at residues Ser-172, Ser-300, Tyr-334 in both WT and

130 PYK2-deficient cells (Fig. 2f, Supplementary Fig. 4a, b). Interestingly, we could only detect

131 Y171 phosphorylation in WT cells (Fig. 2f, g Supplementary Fig. 4b), while S56 and Y312 residues were modified in PYK2-deficient cells (Fig. 2f, Supplementary Fig. 4a, b), possibly reflecting on modification by other enzymes. In fact, Src tyrosine kinase Lyn was capable of

134 phosphorylating IRF5 at orthologues of Y312 and Y334 sites in in vitro co-expression 135 system $^{19}$. We individually mutated these sites (Y171, Y312, Y334) as well as the published 
136 Y104 site ${ }^{31}$ into phenylalanine residues and explored the consequence of these mutations in the

137 above-mentioned reporter and in vitro phosphorylation assays (Supplementary Fig. 1a, f).

138 The Y172F mutant of human v2 IRF5 (Y171 of mouse IRF5) and the double mutant Y172,

139 S173A (Y171, S172 of mouse IRF5) (Fig. 2h) both showed diminished ability to activate the

140 TNF-luciferase reporter in the presence of PYK2, whereas the Y104F, Y329F (mouse Y312)

141 and Y351F (mouse Y334) mutations had no inhibitory effect (Fig. 2h). Similarly, we observed

142 reduction in PYK2-dependent phosphorylation of IRF5 Y172 and Y172/S173 but not other

143 IRF5 mutants (Fig. 2i). Together, these results indicate that PYK2 mediates LPS-induced

144 activation of IRF5, by phosphorylating the tyrosine site Y172 (mouse Y171) of IRF5.

146 Recently, specific inhibitors of PYK2 and a related kinase FAK have been developed ${ }^{32}$. One

147 of them called defactinib (also known as VS-6063 or PF-04554878), with high selectivity to

148 PYK2 and FAK1 and low affinity to kinases outside the family ${ }^{33}$, has been successfully used

149 to tackle cancer in a mouse model and is currently being tested in clinical trials ${ }^{34,35}$. Here we

150 explored the effect of defactinib on IRF5 activation and IRF5-dependent gene expression by

151 macrophages. We first established the concentration range of defactinib well tolerated by

152 RAW264.7 macrophages (Supplementary Fig. 5a). At such concentrations $(0.3-1 \mu \mathrm{M})$, it

153 reduced LPS induced PYK2 phosphorylation (Supplementary Fig. 5b) and effectively

154 inhibited TNF reporter activity and gene expression in RAW264.7 macrophages in a dose

155 dependent manner (Supplementary Fig. 5c, d). Moreover, defactinib inhibited TNF reporter

156 activity in wt and IRF5-deficient RAW264.7 macrophages in which IRF5 expression was

157 restored via ectopic expression of IRF5, but not in PYK2-deficient cells (Fig. 3a), suggesting

158 that defactinib acts in an IRF5- and PYK2-specific manner. PF-573228 inhibitor with described

15950 - 250-fold higher selectivity for FAK over PYK2 ${ }^{36}$, also reduced TNF reporter activity but

160 at a higher concentration than defactinib (Supplementary Fig. 5e). We speculated that PF- 
161573228 may be targeting PYK2 in this system, and indeed observed no further inhibition in

162 PYK2-deficient cells (Supplementary Fig. 5e). The residual LPS-induced TNF reporter

163 activity in cells treated with defactinib and PYK2 deficient cells (Fig. 2c) is consistent with the

164 involvement of other PYK2 independent pathways in control of the TNF gene. As NF- $\mathrm{BB}$ is a

165 known critical regulator of $T N F^{37}$, we examined NF-kB activation in the cells deficient in

166 PYK2 and/or treated with defactinib. Contrary to the results obtained in RAW264 cell lines

167 stably expressing shRNA of $\mathrm{PYK}^{28}$, we observed little effect on the p65/RelA

168 phosphorylation and IkBa degradation in polyclonal RAW264 cell populations with CRISPR-

169 Cas9 mediated knock-out of PYK2 and/or in cells treated with defactinib (Supplementary Fig.

170 5f). We also detected no reduction in NF-kB reporter activity following treatment with

171 defactinib (Supplementary Fig. 5g). In addition, defactinib prevented LPS-induced nuclear

172 translocation of IRF5 but not p65/RelA (Fig. 3b), ruling out a major role for NF-אB in PYK2

173 signalling pathway in these cells.

174 Mirroring our PYK2 deficiency data in RAW264.7 macrophages (Fig. 2), LPS-induced IRF5 and RNA polymerase II recruitment to its target promoters was suppressed in primary mouse bone marrow derived macrophages (BMDMs) treated with defactinib (Fig. 3c). The expression

177 of some pro-inflammatory cytokines and chemokines was also effectively suppressed by

178 defactinib (Fig. 3d, Supplementary Fig. 6b), without affecting cell viability (Supplementary

179 Fig. 6a). Similar results were obtained in macrophages in response to activation of C-type

180 lectin receptor Dectin-1 pathway, in which IRF5 has been shown to play a key role ${ }^{38}$, with

181 WGP (dispersible whole glycan particles) (Supplementary Fig. 6c).

182 To investigate the global impact of PYK2 inhibition on IRF5 target gene expression, we 183 compared LPS-induced transcriptomes in WT and IRF5 KO BMDMs treated with either 184 defactinib or vehicle. Principle component analysis (PCA) of differentially expressed genes 185 (DEGs) $(\mathrm{p}<0.05)$ clearly separated WT and IRF5 KO, as well as untreated and LPS-treated 
sample groups (Fig. 3e). LPS stimulated WT samples treated with defactinib or vehicle were also clearly separated, with the defactinib treated samples grouping closely with the IRF5 $5^{-/}$.

188 Conversely, defactinib had very little effect on IRF5 ${ }^{-/}$cells stimulated with LPS (Fig. 3e). This 189 was reflected in the number of DEGs: 4,026 for WT and only 217 for IRF5 ${ }^{-/}$BMDMs treated $^{-}$ with defactinib at $2 \mathrm{~h}$ of post LPS stimulation (Fig. 3f, Supplementary Fig. 6d). Gene ontology

191 (GO) analysis for defactinib down-regulated genes revealed that they are predominantly proinflammatory in nature (e.g. cellular response to interferon-beta, regulation of inflammatory response, cytokine activity etc). These GO terms were also enriched in LPS induced genes, and in IRF5 up-regulated genes, suggesting that defactinib is highly specific for IRF5 target genes ${ }^{22}$ (Fig. 3g). We next investigated the correlation between IRF5 regulated genes and defactinib target genes. The majority of IRF5 up-regulated genes were strongly repressed by defactinib and there was a high degree of overlap between IRF5 up- and defactinib down- regulated genes, including Illa, Illb, Il6, Il12a, Il12b, Il23a, Ccl3, Ccl4 etc (Fig. 3h, Fig. Supplementary Fig. 6b). Interestingly, there was also a smaller overlap between IRF5 down-regulated genes and defactinib up-regulated genes, suggesting that the actions of IRF5 and defactinib are in direct opposition to each other. Similar to our finding in mouse BMDMs, we saw robust inhibition of LPS-induced expression and production of IRF5-dependent cytokines in human monocyte derived macrophages treated with defactinib at 0.5-5 $\mu \mathrm{M}$ concentrations, which did not affect cell viability (Supplementary Fig. 7a, b, c). Taken together, PYK2 inhibition suppresses IRF5-dependent innate sensing and inflammatory response in both mouse and human macrophages.

IRF5 activity in mononuclear phagocytes (MNPs) plays a critical role in the pathogenesis of intestinal inflammation and that mice deficient in IRF5 are protected from overblown colitis ${ }^{8,9}$.

210 Here we explored if inhibition of Pyk2 would also improve the intestinal immunopathology in

211 a model of Helicobacter hepaticus infected and anti-IL-10R monoclonal antibodies

212 administered (Hh+anti-IL10R) colitis, which is characterised by IL-23-dependent intestinal inflammation along with a robust $\mathrm{T}$ helper type 1/type 17 (Th1/Th17)-polarized effector T cell

214 response $^{39}$ (Supplementary Fig. 8a). As expected, Hh+anti-IL10R-infected mice developed 
215 inflammation in the colon after a week. However, intestinal pathology, as well as immune cell

216 infiltrate, and PYK2 activation in colon tissue were reduced in the animals, which received

217 defactinib (Fig. 4a, b, c; Supplementary Fig. 8b, c). We also observed attenuated induction 218 of Illa, Illb, Il6, Tnf, Il12b, Ccl4 and other pro-inflammatory cytokines and chemokines ${ }^{40}$ in 219 the colon of defactinib-treated Hh+anti-IL-10R infected animals (Fig. 4d). To examine if the 220 observed reduction in cytokine expression reflected on a lower number of monocytes 221 infiltrating the colon or was related to their intrinsic reprogramming, we also analysed gene expression in total colonic leukocytes and isolated monocyte/macrophages. Interestingly, the downregulation of $I l 6, T n f, I 12 b$ and $C c l 4$ expression in response to defactinib was detected in (1) colon tissue, (2) total colonic leukocytes and (3) isolated colonic monocytes and macrophages, while the reduction of $C c l 5$ expression and an upward trend in $I l 10$ expression was only observed in isolated macrophages, indicating that production of these mediators by other cells may mask the effect of IRF5 pathway inhibition in macrophages (Supplementary Fig. 8d).

Next, we tested the impact of PYK2 inhibition in biopsies derived from the colonic mucosa of patients with active ulcerative colitis by measuring cytokine production at concentrations not affecting cell viability in these samples (Supplementary Fig. 9 a, b). We found elevated cytokine production in biopsies obtained from the sites of active inflammation in comparison to those from adjacent non-inflamed colon. Incubation with increasing doses of defactinib significantly lowered IL-6 and IL-12p70, identified as part of a cassette of inflammatory molecules that mark anti-TNF $\alpha$-resistant $\operatorname{IBD}^{41}$ (Fig. 4e). We also observed a trend towards increased IL-10 production by the defactinib treated biopsies, but production of IL-1 $\beta$ appeared to be unaffected. Therefore, pharmacological inhibition of PYK2 effectively dampens intestinal inflammation, positioning defactinib and related PYK2 inhibitors as attractive molecules for repurposing to treat patients with UC.

In conclusion, we propose the following pathway involving Pyk2 and IRF5 in macrophages.

243 Upon TLR4 stimulation by LPS or Dectin-1 stimulation by glucan, PYK2 is activated by 
244 phosphorylation at Tyr-402 28 . MyD88 is likely to be the essential linking adaptor between

245 TLRs and PYK2/IRF5 complex as earlier studies have shown impairment of PYK2 activation

246 in TLR ligand treated MyD88-deficient cells ${ }^{28}$. PYK2 autophosphorylation has been suggested

247 to occur with the help of Src and possibly other kinases ${ }^{42,43}$. The recruitment of PYK2 to IRF5

248 upon Dectin-1 stimulation is likely to be Syk-dependent ${ }^{44}$. We show that PYK2 phosphorylates

249 IRF5 on site Tyr-171 (mouse) contributing to its activation and transcription of pro-

250 inflammatory cytokines (Supplementary Fig. 10). Multiple sites of phosphorylation detected

251 in IRF5, both serine and tyrosine, highlight the complexity of IRF5 activation and multiplicity

252 of signalling pathways ${ }^{11,19,45,46}$. Yet, a clear mechanistic interaction between two established

253 IBD risk genes, PYK2 and IRF5, in macrophages, identified in this study, combined with an

254 acceptable toxicological profile of PYK2 inhibitor defactinib shown in cancer clinical trials ${ }^{47}$,

255 deserves a closer look from the therapeutic perspective. We propose that defactinib is an

256 attractive molecules for repurposing to treat patients with ulcerative colitis ${ }^{8,9}$, and with other

257 inflammatory conditions, such as $\operatorname{arthritis}^{40,48}$ acute lung injury ${ }^{40}$ and atherosclerosis ${ }^{49}$, in

258 which IRF5 function in macrophages has been intimately linked to pathogenicity. It may even

259 prove useful in dampening lung inflammation in the severe COVID 19 patients, whose lungs

260 are filled with monocyte-derived macrophages expressing high levels of IRF molecules,

261 including IRF5 50 . 


\section{Acknowledgements:}

263 We are grateful to Dr Jelena Bezbradica-Mircovic (University of Oxford) for critical reading

264 of the manuscript and useful comments. This work was supported by the Versus Arthritis (PhD

265 studentship 209966 to HA), the BRC3 Gastroenterology and Mucosal Immunology (ALC and

266 ST) and the Wellcome Trust (Investigator Award 209422/Z/17/Z to IAU). We thank GSK for

267 providing their published kinase inhibitor library for this project. 
Methods

\section{Reagents.}

Animals. Mice were bred and maintained under SPF conditions in accredited animal facilities at the University of Oxford. All procedures were conducted according to the Operations of

272 Animals in Scientific Procedures Act (ASPA) of 1986 and approved by the Kennedy Institute of Rheumatology Ethics Committee. Animals were housed in individually ventilated cages at a constant temperature with food and water ad libitum. C57Bl/6 mice were purchased from the University of Oxford BMS.

Cell culture. RAW264.7 and 293 TLR4/CD14/MD-2 cells were cultured in DMEM (Lonza) supplemented with $10 \%$ FBS (Gibco) and $1 \%$ Pen/Strep (Lonza). Bone marrow cells were extracted from wild type mice and cultured with recombinant GM-CSF (20ng/mL; Peprotech).

On day 8, adherent cells were replated, and stimulated with either LPS (100ng/mL, Enzo) or whole glucan particles (100 $\mu \mathrm{g} / \mathrm{mL}$, Invivogen $)$.

Human monocytes were isolated from leukocyte cones of healthy blood donors. Peripheral blood mononuclear cells (PBMC) were enriched by Ficoll gradient. Monocyte-derived macrophages were generated using adherence method selection and GM-CSF differentiation. Whole PBMC $\left(50 \times 10^{6}\right)$ were plated in RPMI-1640 medium for 90 min. After 2 washes with PBS, adherent monocytes were differentiated into macrophages over a 5 days in the presence of $50 \mathrm{ng} / \mathrm{mL}$ GM-CSF (Peprotech) in RPMI supplemented with 10\% foetal calf serum (FCS) (Sigma-Aldrich), $100 \mathrm{U} / \mathrm{mL}$ penicillin, $100 \mathrm{mg} / \mathrm{mL}$ streptomycin, $30 \mathrm{mM}$ HEPES, and 0.05 mM $\beta$-mercaptoethanol.

290 Hoxb8 macrophage progenitors were a gift from the Sykes Lab (Harvard Medical School). 291 Progenitors were cultured in RMPI-1640 medium (Lonza) supplemented with 10\% FBS 292 (Gibco), $\beta$-mercaptoethanol (30 mM; Life Technologies), recombinant GM-CSF (10ng/ml; 
293 Peprotec) and $\beta$-estradiol ( $1 \mu \mathrm{M}$; Sigma-Aldrich). To differentiate into macrophages,

294 progenitors were washed three times with RPMI 1640 medium to remove the $\beta$-estradiol and

295 incubated in complete RPMI 1640 medium supplemented with $10 \%$ heat-inactivated FBS,

$29630 \mathrm{uM} \beta$-mercaptoethanol, and $20 \mathrm{ng} / \mathrm{mL}$ GM-CSF and incubated for 4 days. All cells were

297 incubated in a $5 \% \mathrm{CO}_{2}$ humidified atmosphere at $37^{\circ} \mathrm{C}$.

298

299

300 RNA extraction and Quantitative Real-Time PCR. Total RNAs were isolated from cells

301 using RNeasy Mini Kit (Qiagen) and reverse transcribed to cDNA using High-Capacity cDNA

302 Reverse Transcription Kit (Life Technologies) as per the manufacturer's protocol. RNA from sorted cells was isolated utilising the RNeasy Micro kit (Qiagen). Real-time PCR reactions were performed on a ViiA7 system (Life Technologies) with Taqman primer sets for Hprt, using the comparative $\mathrm{Ct}(\Delta \Delta \mathrm{Ct})$ method and normalised against Hprt levels or RPLPO levels

307 for mouse or human, respectively.

\section{RNA-Sequencing analysis.}

310 Libraries were sequenced on Illumina HiSeq4000 yielding $>40 \times 10^{6} 150$ b.p. paired end reads

311 per sample. These were mapped to the mm10 genome using STAR ${ }^{51}$ with the options: "--

312 runMode alignReads --outFilterMismatchNmax 2." Uniquely mapped read pairs were counted

313 over annotated genes using featureCounts ${ }^{52}$ with the options: “-T 18 -s 2 -Q 255." Differential

314 expression was then analysed with DESeq $2^{53}$ and genes with fold changes $>2$ and false 315 discovery rates (FDRs) $<0.05$ were deemed to be differentially expressed. Variance stabilised

316 (VST) counts for all DESeq2 differentially expressed genes, likelihood ratio test, false 317 discovery rates $($ FDRs $)<0.05$, were used for dimensionality reduction. For direct comparisons 
genes with fold changes $>2$ and FDR $<0.05$ were deemed to be differentially expressed. Gene set enrichment analysis was performed using one-sided Fisher's exact tests (as implemented in the 'gsfisher' R package https://github.com/sansomlab/gsfisher/). RNA sequencing data that support the findings of this study have been deposited in GEO with the accession code GSE141082.

Measurement of cytokine production. Mouse serum cytokine concentrations were analysed by ELISA (Mouse IL12p70, \#DY419-05), and Cytometric Bead Array (IL6 \#558301, IL1 $\beta$ \#560232, BD Biosciences) as per manufacturer's instructions. IL-1 $\beta$, IL-6, and IL12p70 concentration in Human biopsy or cell culture supernatants was measured by Cytometric Bead Array (IL1ß\#558279, IL6\# 558276, IL12p70\#558283). IL-10 concentration in human intestinal biopsy supernatants was measured by ELISA ( \#DY217B-05, R\&D systems). TNF $\alpha$ was measured in human monocyte-derived macrophage culture supernatants by ELISA (\#DY210-05, R\&D systems). All cytokine detection was performed according to manufacturer's instructions.

Western blots. Cells were lysed in 1\% TX-100 lysis buffer (1\% v/v TX-100, 10\% v/v glycerol, $1 \mathrm{mM}$ EDTA, $150 \mathrm{mM} \mathrm{NaCl}, 50 \mathrm{mM}$ Tris $\mathrm{pH}$ 7.8) supplemented with protease inhibitor cocktails (Roche). Lysates were incubated on ice for $30 \mathrm{~min}$ and cleared by centrifugation at

$33813,000 \mathrm{rpm}$ for $10 \mathrm{~min}$ at $4^{\circ} \mathrm{C}$. Protein quantification was performed with the Qubit assay 339 (Thermo Fisher Scientific) according to the manufacturer's protocol. $10 \mu \mathrm{g}$ of lysates were 340 boiled in Laemmli sample buffer (Bio-Rad), resolved on a NUPAGE 4-12\% Bis-Tris gel

341 (Invitrogen), and transferred onto a PVDF membrane (GE Healthcare) by wet western blotting.

342 Membranes were blotted for antibodies for IRF5 (ab21689, Abcam), PYK2 (3292, CST), 
343 Phospho-Pyk2 Tyr402 (3291, CST), alpha-tubulin (3873, CST), Histone H3 (ab1791),

344 GAPDH (ab9485, Abcam) and $\beta$-actin (A5441, Sigma), followed by HRP-conjugated

345 secondary antibodies. Complexes were detected with the chemiluminescent substrate solution

346 ECL (GE Healthcare).

347

348 Subcellular fractionation. Cell pellets were lysed in cytoplasmic lysis buffer $(0.15 \%$ NP-

40, $10 \mathrm{mM}$ Tris $\mathrm{pH} 7.5,150 \mathrm{mM} \mathrm{NaCl}$ ), incubated on ice for 10 minutes, and layered on top of cold sucrose buffer (10 mM Tris $\mathrm{pH} 7.5,150 \mathrm{mM} \mathrm{NaCl}, 24 \% \mathrm{w} / \mathrm{v}$ sucrose). The lysate was centrifuged at $13,000 \mathrm{rpm}$ for 10 minutes at $4^{\circ} \mathrm{C}$ and the supernatant was collected as the cytosolic fraction. The nuclear pellet was lysed in RIPA buffer (150 mM NaCl, 1\% NP-40. $0.5 \%$ Na-DOC, $0.1 \% \mathrm{SDS}, 50 \mathrm{mM}$ Tris $\mathrm{pH} 8.0)$ and sonicated on the Biorupter sonicator (10 cycles of 30 seconds on/30 seconds off), followed by centrifugation at 13,000 rpm for 5 minutes at $4^{\circ} \mathrm{C}$. The supernatant was collected as the nuclear fraction.

Immunoprecipitation. $1 \times 10^{7}$ million cells per immunoprecipitation were seeded and incubated overnight. Media was replaced with serum-free media for $1 \mathrm{hr}$, followed by LPS stimulation at indicated timepoints. Whole cell extracts were prepared with $1 \% \mathrm{TX}-100$ lysis buffer as described above. Lysates were precleared with $100 \mu \mathrm{L}$ TrueBlot Anti-Rabbit Ig IP

361 beads (eBioscience) by rotating. Samples were incubated with $2 \mu \mathrm{g}$ antibody for $2 \mathrm{hr}$, followed

362 by $100 \mu \mathrm{L}$ IP beads (50\% slurry) by rotating overnight. Immunoprecipitates were washed three times with IP wash buffer (1 \% NP-40, $150 \mathrm{mM} \mathrm{NaCl,} 1$ mM EDTA, $20 \mathrm{mM}$ Tris-HCl, pH 8) 
Generation of Pyk2 and IRF5 CRISPR knockouts. 5,000 RAW264.7 cells/well were seeded in 96-well plates and infected the next day with PTK2B (ID:MM0000145196), and IRF5 (ID:MM0000200177) lentiviral particles (pLV-U6g-EPCG) provided by Sigma. Cells were transduced at a multiplicity of infection (MOI) of 10 in medium containing polybrene $(8 \mu \mathrm{g}$ $\mathrm{mL}^{-1}$ ) and spun at $1500 \mathrm{xg}$ for $1 \mathrm{hr}$. After an overnight incubation, media was replaced with fresh media, and selected with $4 \mu \mathrm{g} \mathrm{mL}^{-1}$ puromycin (InvivoGen) for two weeks. Hoxb8 macrophage progenitors were transduced with lentiCas9-v2 lentivirus targeting exon 2 of Irf5 (ID: ENSMUSG00000029771, gRNA ACCCTGGCGCCATGCCACGAGG) and exon3 of PYK2 (ID: ENSMUSG00000059456, gRNA CCCTATTCGCCCACTCAGG). The lentiCas9-v2 plasmid was a gift from Feng Zhang (Addgene plasmid \#52961). Briefly, the lentiCas9-v2 lentivirus were produced from HEK-293FT cells transfected with the lentiCas9v2 plasmid mixed at a 2:1:1 DNA ratio of the lentiviral packaging plasmids pMD2.G (Addgene plasmid \#12259) and psPAX2 (Addgene plasmid \#12260) at a 2:1:1 ratio. Media was replaced 16 hours post-transfection. Two days post transfection, the lentivirus containing mediums were harvested, filtered and added onto Hoxb8 macrophage progenitor cells at a final concentration of $8 \mathrm{ug} / \mathrm{ml}$ polybrene. Transduced cells were allowed to grow for additional four days and selected with 6ug/ml Puromycin for the targeted knockout of Irf5 and Pyk2.

Chromatin Immunoprecipitation. $1 \times 10^{7}$ million cells per ChIP were seeded and incubated overnight. GM-BMDMs cells were pretreated with defactinib or DMSO vehicle for 1 hour, followed by LPS (100 ng/ml) for 2 hours. RAW264.7 cells were stimulated with LPS (500 $\mathrm{ng} / \mathrm{ml}$ ) for 2 hours. Cells were fixed in formaldehyde, quenched with Tris $\mathrm{pH} 7.5$ and washed in PBS. Nuclear lysates were isolated as previously described ${ }^{22}$ and sonicated with a

390 Bioruptor (Diagenode) for 8 cycles (GM-BMDMs) or 10 cycles (RAW264.7). Lysates were 391 immunoprecipitated with $5 \mu \mathrm{g}$ of anti-IRF5 (ab21689; Abcam), anti-RNA Polymerase II 
392 (MMS-128P; Biolegend), or Rabbit Anti-Mouse IgG (ab46540; Abcam). Immunoprecipitated

393 DNA was purified with the PCR Purification Kit (Qiagen). qPCR analysis was carried out in

394 duplicates and represented as \% input. Primer sequences $\mathrm{Illa}$

395 (ACTTCTGGTGCTCATCTGTCATGTT, GCTCTATGGTTCCTGTGTCTGTAGG), Illb

396 (GGATGTGCGGAACAAAGGTAGGCACG, ACTCCAACTGCAAAGCTCCCTCAGC),

397 Il6 (GAGAGAGGAGTGTGAGGCAGAGAGC, GGTTGTCACCAGCATCAGTCCCAAG),

Ill2b (GCAAGGTAAGTTCTCTCCTCTTCCC, AATGACTATTTGAAGCCCCTGTCGT),

TNF (GCTAAGTTCTTCCCCATGGATGTCCC,

Kinase inhibitors screening and luciferase reporter assay. RAW264.7 cells were seeded in eighteen 96-well plates at 50,000 cells/well a day before transfection as described above. $1 \mathrm{hr}$ prior to LPS treatment, cells were treated with $10 \mathrm{uM}$ of inhibitors in quadruplicates. For experiment wells ( $\mathrm{n}=4$ for each inhibitor set AG-AK, total amounts for 16 plates (AG-AJ1-4) - 80 wells per plate and 2 plates (AK1,2-3,4)- 96 wells per plate) $21 \mathrm{ml}$ of Opti-Mem (Gibco) was mixed with DNA: $50 \mu \mathrm{g}$ of pBent-HA-IRF5, $50 \mu \mathrm{g}$ of pGL3-5'3TNF-luc and $25 \mu \mathrm{g}$ of pEAK8-Renilla, vectors described in ${ }^{21,54} .5 \mathrm{ml}$ of Opti-Mem mixed with $200 \mu \mathrm{l}$ of Plus reagent was added to the DNA solution and incubated for 5-15 min. Then, $5 \mathrm{ml}$ of Opti-Mem was mixed with $500 \mu$ of Lipofectamine LTX reagent, added to the DNA/Plus solution and incubated for

$41130 \mathrm{~min}$. For controls (amount for 4 plates - two 4 well-rows each), $800 \mu$ l of Opti-Mem was

412 mixed with DNA: $2 \mu \mathrm{g}$ of pBent-HA-IRF5 or pBent2-empty, $2 \mu \mathrm{g}$ of pGL3-5'3'-TNF-luc and

$4131 \mu \mathrm{g}$ of pEAK8-Renilla. $200 \mu \mathrm{l}$ of Opti-Mem mixed with $8 \mu \mathrm{l}$ of Plus reagent was added to the

414 DNA solution and incubated for 5-15 min. Then, $200 \mu$ of Opti-Mem was mixed with $20 \mu \mathrm{l}$ 415 of Lipofectamine LTX reagent, added to the DNA/Plus solution and incubated for $30 \mathrm{~min}$. To 416 transfect cells, $20 \mu \mathrm{l}$ of the DNA/transfection reagent mix was added per well. Next day, cells 
were pre-incubated for $1 \mathrm{hr}$ with $20 \mu \mathrm{l}$ of inhibitor (or 1\% DMSO to control wells) in serum-

418 free DMEM (final conc. $0.1,1$ or $10 \mu \mathrm{M}$ in $1 \%$ DMSO). Then, $1 \mu \mathrm{g} / \mathrm{ml}$ of LPS was added to

419 the cells and 6 hours later the culture medium was and the plate-bound cells were kept frozen

420 (-20 C). Cells were lysed using the Dual-Glo Luciferase Assay kit (Promega) according to the

421 manufacturer's protocol and analysed in a FLUOstar Omega microplate reader (BMG

422 Labtech). Raw firefly luciferase activities (or values normalized against Renilla luciferase activities) in wells incubated with the kinase inhibitors were divided by the luciferase activity values in the control wells (DMSO vehicle only, cells expressing IRF5 and stimulated with LPS) and expressed as part of a whole or a percentage of IRF5 reporter activity (which was 1 or $100 \%$ in cells treated with DMSO only).

427

Kinase assays.

293 ET cells were plated at 250,000 cells/well in six-well plates and a day later were transfected with $1 \mu \mathrm{g}$ of pBent2-HA-IRF5 and $1 \mu \mathrm{g}$ of plasmid encoding one of the myc-tagged candidate IRF5 kinases (in the pEAK8-myc vector) using Lipofectamine2000 ${ }^{\mathrm{TM}}$ (Life technologies) according to the manufacturers protocol. The cell lysates were subjected to kinase assays using a modification of an established protocol ${ }^{55}$. Cells were washed in PBS and lysed on the ice in kinase reaction buffer (20 mM HEPES pH 7.5, 137 mM NaCl, 0.5 mM EGTA, 25 mM MgCl2, $0.2 \%$ Triton X-100, $10 \%$ Glycerol) with added protease (EDTA-free! complete-mini protease inhibitor cocktail, Roche, \#11836170001) and phosphatase inhibitors (phosphatase inhibitor cocktail II, Sigma, \#P5726). 2 mM of TCEP (\#C4706, Sigma), 1mM of GTP (\#G8877, Sigma) and $50 \mu \mathrm{M}$ S- $\gamma$-ATP (ab138911, Abcam) was added sequentially to lysing samples. The reactions were mixed by vortexing and kept an a rocking surface for $1 \mathrm{~h}$ at $37 \mathrm{C}$. The reactions

440 were stopped by adding $50 \mathrm{mM}$ EDTA and moving them on ice. p-Nitrobenzyl mesylate 441 (PNBM, ab138910, Abcam) was dissolved in DMSO to $50 \mathrm{mM}$. PNBM working solution was 
prepared by adding $5 \mu \mathrm{L}$ of deionized water five times mixing after each addition to $25 \mu \mathrm{L}$ of the PNBM stock, and then was added 1/10 to kinase reactions (at $2.5 \mathrm{mM}$ ), which were further incubated for $2 \mathrm{hrs}$ at room temperature. The bulk of the reactions were subjected to immunoprecipitations using anti-thiophosphate-ester antibody $(1 \mu \mathrm{g}$ per reaction, ab92570, Abcam) and Protein G Sepharose 4 Fast Flow Media (\#11524935, GE Healthcare) to pulldown phosphorylated IRF5. The rest of the reactions and the pull-downs were mixed with SDS PAGE loading buffer and subjected to SDS PAGE following Western blotting.

Mass spectrometry analysis. IRF5 was immunoprecipitated from LPS-stimulated WT and PYK2 KO RAW264.7 cells ( $5 \times 10^{7}$ cells) as described in immunoprecipitation section. Eluents were subjected to in-solution digestion as described previously ${ }^{56}$. In brief samples were reduced and alkylated before double precipitation with Chloroform/Methanol as described ${ }^{57}$. Protein pellets were resuspended in $50 \mu \mathrm{L} 6 \mathrm{M}$ urea for solubilisation. The samples were diluted to $1 \mathrm{M}$ Urea in $100 \mathrm{mM}$ Tris buffer for tryptic digest. Following overnight digestion, peptides were acidified with 3\% Formic acid and desalted with solid phase extraction Sola cartridges (Thermo). Peptides were eluted with $600 \mathrm{uL}$ glycolic acid solution (1M glycolic acid, 80\% acetonitrile, $5 \%$ trifluoroacetic acid). Phospho-peptide enrichment was performed using a $\mathrm{TiO}_{2}$ protocol as described ${ }^{58}$ with eluates from the Sola cartridges adjusted to $1 \mathrm{~mL}$ with $1 \mathrm{M}$ glycolic acid solution and incubated for 5 minutes with $50 \mathrm{uL} \mathrm{TiO}_{2}$ bead slurry solution. Bead washes (200 uL) were carried out as previously described. In short, beads were sequentially washed with $200 \mathrm{uL}$ glycolic acid solution, ammonium acetate solution (100 $\mathrm{mM}$ ammonium acetate in $25 \%$ acetonitrile) and $10 \%$ acetonitrile solution repeated in triplicate. Phospho-peptides were eluted, following incubation for 5 minutes at room temperature with $50 \mathrm{ul}$ ammonia solution $(5 \%)$ and centrifuged, this was repeated in triplicate. The three eluate fractions were combined and dried using a SpeedVac and pellets were stored at $-80^{\circ} \mathrm{C}$ until analysis. For analysis by 
nano-liquid chromatography tandem mass spectrometry (nLC-MS/MS), a Dionex UHPLC system coupled to an Orbitrap Fusion Lumos mass spectrometer was used as described previously ${ }^{59}$. Raw MS files were subjected to processing using PEAKS (version 8.5) software and searched against the UniProtSP Mus Musculus database. Searches included the data refine, denovo PEAKS and PEAKS PTM modes, the latter of which included phosphorylation on Ser (S), Thr (T) and Tyr (Y) residues. The proteomics data and MS raw files have been deposited to Proteome Xchange Consortium via the $\operatorname{PRIDE}^{60}$ partner repository with the dataset identifier PXD014033 (https://www.ebi.ac.uk/pride/archive/).

Helicobacter hepaticus- induced colitis model. Mice were free of known intestinal pathogens and negative for Helicobacter species. Animals from each experimental group were cohoused. On days 0,1 , and 2 , mice were injected i.v. with $1 \mathrm{mg} / \mathrm{kg}$ defactinib or vehicle $(5 \% \mathrm{DMSO}$, 2.5\% Solutol HS (Sigma), 2.5\% absolute ethanol, 90\% Dulbecco’s PBS). Daily, starting from day 3 , mice were injected i.p. with $5 \mathrm{mg} / \mathrm{kg}$ defactinib or vehicle. 30 minutes after the initial i.v. injection, mice were infected with $1 \times 10^{8}$ colony forming units Hh on days 0 and 1 by oral gavage with a 22G curved, blunted needle (Popper \& Sons). Mice were injected intraperitoneally once on day 0 with $1 \mathrm{mg}$ anti-IL10R blocking antibody (clone 1B1.2). Infected mice were monitored daily for colitis symptoms. Mice were culled one week after day of infection, and organs were harvested for analysis.

487 Isolation of lamina propria leukocytes. Colons and/or caeca were harvested from mice, washed in PBS/BSA and content flushed with forceps. Intestines were then opened longitudinally and washed once more before blotting to remove mucus. Gut tissue was then cut into $1 \mathrm{~cm}$ long pieces and placed in $50 \mathrm{~mL}$ centrifuge tube (Greiner) in ice cold PBS $+0.1 \%$ 
492 Streptomycin (PS, Lonza) $+5 \mathrm{mM}$ EDTA (Sigma-Aldrich) at $37{ }^{\circ} \mathrm{C}$ for $10 \mathrm{~min}$ before the 493 supernatant was aspirated. Tissue was placed in $40 \mathrm{~mL}$ PBS $+0.1 \% \mathrm{BSA}+1 \%$ PS for $5 \mathrm{~min}$. 494 Intestines were then incubated with $20 \mathrm{~mL} \mathrm{RPMI}+10 \% \mathrm{FCS}+1 \% \mathrm{PS}+2.5 \mathrm{U} / \mathrm{mL}$ Collagenase 495 VIII (Sigma-Aldrich) + 2 U/mL DNAse I (Roche), shaking at $200 \mathrm{rpm}$ for $45 \mathrm{mins}-1$ hour at $49637^{\circ} \mathrm{C}$. Supernatant was filtered through a $70 \mu \mathrm{m}$ cell strainer to which $30 \mathrm{~mL}$ of ice cold PBS $497+0.1 \%$ BSA $+1 \%$ PS +5 mM EDTA was added to ablate collagenase/DNase activity. Cells 498 were washed in $30 \mathrm{~mL}$ PBS/BSA before filtering once more through a $40 \mu \mathrm{m}$ cell strainer. The 499 cells were then pelleted by centrifugation at $400 \mathrm{rcf}$ for 10 minutes at $4{ }^{\circ} \mathrm{C}$ and resuspended in $5001 \mathrm{~mL}$ RPMI $+10 \%$ FCS $+1 \%$ PS before counting.

502 Flow cytometry. CBA quantification of cytokine levels were performed on a FACSCanto II 503 (BD) and analysed using Flowjo (Treestar Inc.). Acquisition of mouse samples was performed using either LSR II or Fortessa X20 flow cytometers with FACSDiva (BD), followed by analysis in Flowjo (Treestar Inc.). Gating strategy in Fig. S8e.

507 Extracellular labelling of cells. $5 \times 10^{5}-2 \times 10^{6}$ cells were plated on U-bottom 96 well plates.

508 Cells were washed twice with $150 \mu \mathrm{L}$ FACS buffer (PBS $+0.1 \%$ BSA +1 mM EDTA $+0.01 \%$ 509 Sodium Azide) at $400 \mathrm{rcf}$ for $3 \mathrm{~min} 4{ }^{\circ} \mathrm{C}$. Cells were then $\mathrm{Fc}$ blocked for 10 min with $510 \alpha \mathrm{CD} 16 / \mathrm{CD} 32$ (BD) $1 / 100$ in $20 \mu \mathrm{L}$ FACS buffer at room temperature (RT) followed by 511 washing once in $150 \mu \mathrm{L}$ FACS buffer. Fixable Viability Dye eFluor ${ }^{\circledR} 780$ (ThermoFisher) and 512 primary extracellular antibodies (Table 1.1) were added for $30 \mathrm{~min}$ at $4{ }^{\circ} \mathrm{C}$ in $20 \mu \mathrm{L}$ FACS 513 buffer in the dark. Labelled cells were then washed twice with $150 \mu \mathrm{L}$ FACS buffer. Cells were 514 then fixed for 30 mins in $50 \mu \mathrm{L}$ Cytofix (BD), washed twice with $150 \mu \mathrm{L}$ FACS buffer, and 515 resuspended in $200 \mu \mathrm{L}$ FACS buffer before acquisition. 


\begin{tabular}{|l|l|}
\hline \multicolumn{2}{|l|}{ Table.1.1 Antibodies used in flow cytometry analysis } \\
\hline Antigen & Clone \\
\hline CD45 & $30-$ F11 \\
\hline CD11b & M1/70 \\
\hline CD11c & N418 \\
\hline Siglec F & E50-2440 \\
\hline Ly6G & 1 A8 \\
\hline F4/80 & BM8 \\
\hline CD103 & $2 \mathrm{E} 7$ \\
\hline CD206 & CO68L2 \\
\hline MHC II & M5/114.15.2 \\
\hline CD19 & 6 D5 \\
\hline CD138 & $281-2$ \\
\hline NK1.1 & PK136 \\
\hline CD3e & $145-2 C 11$ \\
\hline TCRgd & TER-119 \\
\hline Ter119 & \\
\hline
\end{tabular}

518 FACS sorting. Colon lamina propria cells were prepared as described above. A small aliquot

519 of each sample was stored in RNAlater (Sigma Aldrich) for further processing. Two to three

520 samples were pooled in order to gain sufficient numbers for sorting. The cells were labelled as

521 described above with the antibodies in Table 1.1, except no fixation step was performed.

522 Labelled cells were washed twice with $1 \mathrm{~mL}$ FACS buffer and resuspended in 500uL FACS

523 buffer containing DNAse I (10ug/mL, Roche). Sorting of the cells was performed into 500uL

524 RNAlater on FACSAria III (BD Biosciences) at the Kennedy Institute of Rheumatology FACS

525 facility. 
526 Culture of UC patient colonic mucosal biopsies. Intestinal pinch biopsies were obtained

527 from Ulcerative Colitis patients registered in the Oxford IBD Cohort, attending the John

528 Radcliffe Hospital Gastroenterology Unit (Oxford, UK). This cohort comprises 1896 patients

529 with UC, median age 31 at diagnosis, treated with biological therapy (23\%) or conventional

530 steroids/immunomodulators (77\%) for active disease, in addition to mesalazine. Biopsies

531 were collected during routine endoscopy. Informed, written consent was obtained from all

532 donors. Human experimental protocols were approved by the NHS Research Ethics System

533 (Reference numbers: 16/YH/0247). Biopsies were washed in PBS and transferred into wells

534 containing RPMI-1640 + 10\% FCS $+20 \mu \mathrm{g} / \mathrm{mL}$ G418 (Thermo Fisher) $+20 \mathrm{U} / \mathrm{mL}$ Pen/Strep

535 and cultured for 24 hours.

536

537 UC biopsy viability assessment. Biopsies were fixed in 4\% PFA in PBS (\#30525-89-4,

538 Santa Cruz) for $24 \mathrm{hrs}$ at RT and transferred to $70 \%$ ethanol. Fixed biopsies were then

539 dehydrated and embedded in paraffin blocks, and $5 \mu \mathrm{m}$ sections were cut. Embedding and

540 sectioning of tissues was carried out by the Kennedy Institute of Rheumatology Histology

541 Facility (University of Oxford). Viability of intestinal biopsies was measured by TACS®

542 TdT in situ (Fluorescein) TUNEL assay (\#4812-30-K, R\&D systems) according to

543 manufacturer's instructions. Sections were then mounted in Glycerol Mounting Medium with

544 DAPI and DABCO (\#ab188804, Abcam) and cover-slipped. Images of three non-sequential

545 sections per sample were acquired. Three images per section were acquired at 20x

546 magnification using a BX51 microscope (Olympus). To generate the apoptotic index, the

547 total cell number was enumerated by counting DAPI ${ }^{+}$, and TUNEL(FITC) ${ }^{+}$cells in ImageJ,

548 and calculating the percentage of total cells that were TUNEL ${ }^{+}$. 
550 Histopathological assessment. Post-sacrifice, $0.5 \mathrm{~cm}$ pieces of caecum, and proximal, mid

551 and distal colon were fixed in PBS $+4 \%$ paraformaldehyde (Sigma Aldrich). Fixed tissue

552 was embedded in paraffin blocks, and sectioned using a microtome and stained with

553 Haematoxylin and Eosin (H\&E) by the Kennedy Institute of Rheumatology Histology

554 Facility (Kennedy Institute of Rheumatology, University of Oxford). Sections were scored in

555 a blinded manner by two researchers according to ${ }^{61}$.

556

557 Cell viability. 50,000 cells/well were seeded and incubated overnight. Cell viability was 558 assessed using the Promega CellTiter-Glo ${ }^{\circledR}$ Luminescent kit per the manufacturers protocol 559 and luminescence was measured in a FLUOstar Omega microplate reader (BMG Labtech).

560 Samples were tested in triplicate and normalised to untreated wells.

562 Protein isolation from colon tissue. $1.5 \mathrm{ml}$ Bioruptor Microtubes were filled with $250 \mathrm{mg}$ of

563 Protein Extraction Beads (Diagenode) and filled with RIPA buffer (supplemented with 564 protease and phosphatase inhibitors). $10 \mathrm{mg}$ of tissue was added to the tubes and vortexed 565 briefly. Tubes were sonicated on the Biorupter Pico with $30 \mathrm{sec}$ ON/30 sec OFF for 6 cycles 566 at $4{ }^{\circ} \mathrm{C}$. After each 2 cycles, tubes were vortexed. The supernatant was transferred to a new tube 567 and centrifuged at $13,000 \mathrm{rpm}$ for 10 minutes at $4^{\circ} \mathrm{C}$. The supernatant was transferred to a new 568 tube and $80 \mu \mathrm{g}$ of lysate was used for immunoblot analysis. 
Figure Legends

Figure 1. Small molecule library screening and in vitro validation of shortlisted candidate

\section{IRF5 kinases confirms Pyk2 as a positive hit.}

575

576

577

578

579

580

581

582

583

584

585

586

587

588

589

590

591

592

593

594

595

596

(a) The screening workflow showing the initial large screening in RAW264.7 cells, and the subsequent screens in RAW264.7 and 293 TLR4 cells. The top inhibitors were shortlisted based on their efficacy towards IRF5 reporter and low toxicity. Based on the known activities of these molecules against 221 kinases in the PKIS set, 34 kinases affected by the top inhibitors were shortlisted. The underlined kinases were previously proposed to target IRF5. (b) Impact of the kinases on the IRF5 reporter activity. Luciferase activities were measured in cells coexpressing IRF5 (or empty plasmid control, pBent2), TNF-luc reporter and one of the shortlisted kinases. Reporter activity was calculated as firefly luciferase activity normalised against constitutively expressed Renilla luciferase units and is shown as compared to the values in cells not expressing any kinase. (c) Binding of IRF5 to the shortlisted kinases. Myc-tagged kinases and HA-tagged IRF5 were co-expressed in 293 ET cells. Cell lysates were subjected to immunoprecipitation (IP) using anti-myc antibody and levels of kinases and IRF5 in the IP eluates and proteins were determined by Western blot. Asterisks indicates expected molecular weight. (d) In vitro kinase assays of 293 ET cells co-transfected with HA-IRF5 and myc- or flag-tagged kinases. Proteins in the pull-downs and lysates were detected by Western blotting using antibodies against HA- (IRF5) and myc- and FLAG- (kinases).

\section{Figure 2. PYK2 regulates IRF5 activation and IRF5-mediated transcription}

(a) Endogenous co-immunoprecipitation in RAW264.7 macrophages. Cells were stimulated with LPS for 10 minutes and immunoprecipitated with IRF5, PYK2 or an isotype control antibody. Immunoprecipitates were eluted from IP beads and proteins present in cell lysates (5\% inputs) and eluates were detected by immunoblotting with antibodies against IRF5 or 
PYK2. (b) Immunoblot of LPS-induced PYK2 tyrosine phosphorylation. Blots were probed and PYK2 KO RAW264.7 cells stimulated with LPS 6 hrs or left untreated. (d) IRF5 and pol II binding to Il6 and Ill $a$ gene promoter in resting or LPS-treated (2h, $500 \mathrm{ng} / \mathrm{ml})$ wild type or PYK2 KO RAW264.7 cells as measured by the chromatin immunoprecipitation (ChIP) method. A non-specific IgG antibody was used as a negative control for ChIP. (e) Il6 and Illa mRNA induction in wild type, PYK2 KO or IRF5 KO RAW264.7 cells stimulated with LPS (500 ng/ml) for 0,2 , or $4 \mathrm{hrs}$. Gene expression was measured by qPCR. (f) Phosphorylation sites identified in LPS-stimulated WT and PYK2 KO RAW264.7 cells.

(g) MS/MS spectrum of the IRF5 derived tryptic peptide 152-179 indicating phosphorylation at positions Y171. TLR4 cells and their effect on the TNF-luciferase reporter assay was measured in the absence or presence of PYK2. Reporter activity is expressed as firefly luciferase levels relative to

612 Renilla levels and values are means of three independent experiments. (i) In vitro kinase assay and immunoblot of IRF5- site specific tyrosine mutants. HEKTLR4 cells were co-transfected with FLAG-IRF5 tyrosine mutants as indicated and Myc-PYK2. 10\% lysate was kept for input and the remaining used for in vitro kinase reactions. Kinase assays were detected by western 616 blot using antibodies against Flag-(IRF5) and Myc-(PYK2). All values in (c-e, h) are shown as 617 mean values +/- SEM from $\mathrm{n}=3$ experiments. Comparison by two-way ANOVA $* P<0.05$, $* * P<0.01, * * * P<0.001$, and $* * * * P<0.0001$. 
(a) TNF-luc reporter activity in the absence or presence of ectopically expressed IRF5 in wild shown as means $+/-$ SEM from $n=3$ experiments. Comparison by two-way ANOVA $* * * P<0.001$ and $* * * * P<0.001$. (b) RAW264.7 cells were fractionated into cytosolic and nuclear extracted following $1 \mathrm{~h}$ pre-treatment with defactinib $(1 \mu \mathrm{M})$ and $2 \mathrm{hr}$ stimulation with LPS $(1 \mu \mathrm{g} / \mathrm{ml})$. (c) IRF5 and pol II binding to Il6 and Illb gene promoters in GM-CSF-differentiated mouse BMDMs pre-treated with $3.5 \mu \mathrm{M}$ defactinib (def) or DMSO control and further stimulated with LPS for 2 hrs. Chromatin recruitment was analysed by ChIP. Data are normalized against chromatin amount in lysates (and expressed as percentage of input for each gene) and shown as mean values $+/$ - SEM from $n=3$ individual mice, each performed in duplicates. Comparison by one-way ANOVA $* P<0.05, * * P<0.01$ with multiple test corrections by Tukey. (d) $I l 6$ and $I l 1 b$ expression levels in GM-BMDMs pre-treated with 3.5 $\mu \mathrm{M}$ defactinib (def) or DMSO control

636 for $1 \mathrm{~h}$, followed by LPS stimulation for $2 \mathrm{hrs}$. Data are shown as means +/-SEM for $\mathrm{n}=4$

637 individual mice and analysed by one-way ANOVA $* P<0.05$ and $* * * P<0.001$. (e) PCA analysis of 638 RNA-seq data from WT and Irf5 ${ }^{-/-}$pre-treated with $3.5 \mu \mathrm{M}$ defactinib (def) or DMSO control 639 for $1 \mathrm{~h}$ and further stimulated with LPS for 0 or $2 \mathrm{hrs.} \mathrm{(f)} \mathrm{MA} \mathrm{plots} \mathrm{depicting} \mathrm{effect} \mathrm{of} \mathrm{defactinib}$ 640 on LPS stimulated BMDMs from WT or IRF5-/- mice. Differentially expressed genes (fold 641 change $>1$ and padj $<0.05$ ) are highlighted in red. (g) GO enrichment analysis for differentially expressed genes (as in f). (h) Correlation analysis of IRF5 and defactinib regulated genes after

6432 hrs LPS stimulation. Red indicates genes are differentially expressed (significance as in f) in 644 both comparisons, genes regulated by IRF5 only (black), genes regulated by defactinib only 645 (grey). Venn diagrams demonstrate overlap between IRF5 and defactinib regulated genes. 

colitis and in UC biopsies.

648 (a) H\&E staining of large intestine tissue sections, (b) histology scoring and (c) leukocyte content from $H$ h/anti-IL10R-treated mice, which received either vehicle or defactinib.

(d) Cytokine/chemokine mRNA expression levels in mouse colon tissues from vehicle or defactinib Hh/anti-IL10R treated mice. Data from $(b-d)$ are shown as means $+/-S E M$ for $n=6$ mice $* P<0.05$ and $* * P<0.01$ by unpaired Student's t test. $(\mathbf{E})$ Cytokine proteins levels in biopsies from ulcerative colitis patients from non-inflamed and inflamed tissues treated with defactinib at indicated concentrations per mg of tissue. Data are shown as means +/-SEM for $\mathrm{n}=10$ human donors and analysed by two- way ANOVA where $* P<0.05$ and $* * P<0.01$.

\section{Supporting information}

Source Data 1. Reporter gene assays screening of GSK PKIS set for IRF5 activation inhibitors.

Supplementary Figure 1. Screening and validation assays to identify novel IRF5 kinases left untreated. Data are shown as means +/- SEM for 3 independent experiments each performed in triplicates and analysed by two-way ANOVA where $* P<0.05$ and $* * * * P<0.0001$.

(c) A scheme of small molecule screening for candidate IRF5 kinases. RAW cells were constitutively expressed Renilla luciferase. (1) $24 \mathrm{hrs}$ after transfection cells were pre-treated with a library of inhibitors (four replicate wells per inhibitor) for $1 \mathrm{hr}$ (2) and stimulated with 
671 shown based on activities of the firefly luciferase reporter, raw values or normalised to Renilla

672 luciferase activities to account for non-specific impact of cell viability. Out of 365 molecules,

67357 inhibited IRF5 reporter activity by $30-50 \%, 34-50-70 \%, 8-70-80 \%, 5-80-90 \%$ and 4

674 by $>90 \%$ (the normalised activity readout). (e) Activities of top 10 IRF5 reporter inhibitors are

675 shown where the dataset was analysed based on raw firefly luciferase or normalised to Renilla

676 values. The compounds indicated with numbers are in top 10 independently of normalisation.

677 To calculate reporter activity luciferase values (raw or normalised to Renilla) in wells

678 incubated with kinase inhibitors were divided by the luciferase activity values in the control

679 wells (DMSO vehicle only, cells expressing IRF5 and stimulated with LPS). (f) A scheme of

680 a modified-ATP based IRF5 kinase assay. Cells co-expressing HA-tagged IRF5 with either of

681 the candidate kinases were lysed and incubated with S- $\gamma$-ATP. The newly-produced phosphate

682 groups were further labelled using a reaction with PNBM and the modified proteins were pulled

683 down using anti-thiophosphate ester antibody. (g) Table summarising functional validation of 684 candidate kinases related to Fig. 1b-d.

685

686 Supplementary Figure 2. PYK2 regulates IRF5 activation and IRF5-mediated

687 transcription.

688 (a) Western blot analysis of PYK2 and IRF5 expression in RAW 264.7 cells transfected with 689 control, PYK2 or IRF5 CRISPR-based knockout constructs. (b) Immunoblot analysis for 690 restoring PYK2 expression in PYK2 deficient RAW264.7 cells. (c) TNF-luciferase reported 691 activity in WT and PYK2 KO RAW264.7 cells co-transfected with pBent2-Empty, HA-IRF5, 692 or Myc-PYK2 along with TNF-firefly Luc and pRLTK-Renilla Luc. Cells were stimulated with 693 LPS $(1 \mu \mathrm{g} / \mathrm{ml})$ or left untreated for a further 6 hrs. (d) IRF5 and pol II binding to Tnf gene 694 promoter in resting or LPS-treated (2h, $500 \mathrm{ng} / \mathrm{ml})$ wild type or PYK2 KO RAW264.7 cells as 695 measured by the chromatin immunoprecipitation (ChIP) method. A non-specific IgG antibody 
was used as a negative control for ChIP. Data are normalized against chromatin amount in lysates

697 (and expressed as percentage of input for each gene). (e) Gene expression levels in wild type, PYK2 KO or IRF5 KO RAW264.7 cells stimulated with LPS (500 ng/ml) for 0, 2, or 4 hrs.

699 Gene expression was measured by qPCR. All values in (c-e) are shown as mean values +/-

700 SEM from $\mathrm{n}=3$ experiments. Comparison by two-way ANOVA $* P<0.05, * * P<0.01, * * * P<0.001$, and $* * * * P<0.0001$.

702

Supplementary Figure 3. PYK2 regulates IRF5-mediated transcription in macrophages.

(a) Flow cytometry analysis of HoxB8 cells after 5 days of differentiation with GM-CSF. Day

0 corresponds to cells prior to differentiation. (b) Western blot analysis of IRF5 and PYK2 expression in Hoxb8 macrophage progenitors transfected with PYK2 or IRF5 CRISPR-based macrophages stimulated with LPS (100 ng/ml) 2 hrs. Gene expression was measured by qPCR. Values shown as mean values $+/-$ SEM from $n=3$ experiments. Comparison by two-way ANOVA $* P<0.05, * * P<0.01, * * * P<0.001$, and $* * * * P<0.0001$. phosphorylated sites in IRF5.

(a) MS/MS spectra indicating mouse IRF5 S300, Y334, S445, S56, Y312, S172

715 phosphorylation in LPS-stimulated WT and PYK2 KO cells. Fragmentation ions of the b- and y- series are indicated in blue and red, respectively. (b) Identification of endogenous IRF5

717 phosphorylation sites by tandem mass spectrometry (MS/MS) in LPS-stimulated WT (top panel) and PYK2 KO (lower panel) RAW264.7 cells in which the peptides identified by LCMS/MS are underlined by a blue line. Each line corresponds to a unique MS/MS spectrum.

720 Post-translational modifications including cysteine carbamidomethylation (orange box) and 
721

722

723

724

725

726

727

728

729

730

731

732

733

734

735

736

737

738

739

740

741

742

743

744

745

phosphorylation (red box) are indicated. The location of phosphorylated Tyr 171 (Y171) and Ser172 (S172) observed in WT IRF5 are marked in a red box. (c) IRF5 sites in mouse and equivalent position in human isoform 2.

\section{Supplementary Figure 5. Defactinib affects Pyk2 phosphorylation and IRF5-dependent} gene expression at concentrations that do not affect cell viability.

(a) Cell viability in RAW264.7 cells pre-treated with DMSO/Defactinib for $1 \mathrm{hr}$ followed by LPS $(1 \mu \mathrm{g} / \mathrm{mL})$ for $6 \mathrm{hrs}$. $\mathrm{IC}_{50}$, inhibitor concentration at which $50 \%$ decline in cell viability was observed compared to control (DMSO). (b) Immunoblot of lysates of RAW264.7 cells pretreated for $1 \mathrm{~h}$ with $1 \mu \mathrm{M}$ defactinib (def) or DMSO control, and stimulated with LPS (1 $\mu \mathrm{g} / \mathrm{mL}$ ) for $30 \mathrm{~min}$. Blots were probed with Abs specific for PYK2 phosphorylated on Tyr402, total PYK2 and GAPDH. (c) TNF-luc reporter activity in the absence or presence of ectopically expressed IRF5 in RAW264.7 cells pre-treated for $1 \mathrm{hr}$ with defactinib (or DMSO control) at indicated concentrations followed by LPS (1ug/ml) for $6 \mathrm{hrs}$. (d) Gene expression levels in RAW264.7 cell pre-treated with defactinib (def) or DMSO control for $1 \mathrm{~h}$, followed by LPS stimulation for $4 \mathrm{hrs}$. Data are shown as means $+/-\mathrm{SEM}$ for $\mathrm{n}=3$ and analysed by one-way ANOVA. (e) TNF-luc reporter activity in the absence or presence of ectopically expressed IRF5 in wild type, IRF5 KO and PYK2 KO RAW264.7 cells pre-treated for $1 \mathrm{hr}$ with defactinib (Def), PF-573228 (PF) inhibitor or DMSO control at indicated concentrations and stimulated with LPS $(1 \mu \mathrm{g} / \mathrm{ml})$ for a further 6 hrs. (f) WT and PYK2 KO RAW264.7 cells were pre-treated with $1 \mu \mathrm{M}$ of defactinib (Def) or DMSO vehicle control for $1 \mathrm{~h}$ followed by LPS $(500 \mathrm{ng} / \mathrm{ml})$ at indicated timepoints. Cell lysates were subjected for immunoblot using indicated antibodies. (g) NFkB-luc reporter activity in HEK-TLR4 cells co-expressing IRF5 or empty pBent2 plasmid control. Cells were pretreated with defactinib $(1 \mu \mathrm{M})$ or DMSO control for $1 \mathrm{hr}$ and stimulated with LPS (1 $\mathrm{gg} / \mathrm{ml})$ for $6 \mathrm{hrs}$. Data for (c), (e) and (g) are shown as means +/- SEM 
of three independent experiments, and analysed by 2 way ANOVA. $* * * P<0.001$ and $* * * * P<0.0001$

Supplementary Figure 6. Defactinib affects IRF5-dependent gene expression

(a) Cell viability in GM-BMDMs pre-treated with DMSO/Defactinib for $1 \mathrm{hr}$ followed by LPS defactinib (def) or DMSO control for $1 \mathrm{~h}$, followed by LPS (100 ng/ml) or (c) WGP (100 $\mathrm{ug} / \mathrm{ml}$ ) for $2 \mathrm{hrs}$. Data are shown as means $+/$-SEM for $\mathrm{n}=4$ individual mice and analysed by oneway ANOVA $* P<0.05, * * P<0.01$, and $* * * \mathrm{P}<0.001$. (d) Number of DE genes from RNA-seq.

Supplementary Figure 7. Defactinib affects gene expression in human monocyte-derived macrophages.

(a) Cell viability was measured in human monocyte-derived macrophages (hMDMs) after $3 \mathrm{hrs}$ or 24 hrs of treatment with defactinib. (b) Cytokine mRNA expression levels in human monocyte-derived macrophages pre-treated with defactinib (def, $5 \mu \mathrm{M}$ ) for $1 \mathrm{~h}$ followed by LPS stimulation $(100 \mathrm{ng} / \mathrm{ml})$ for $2 \mathrm{~h}$. Data are shown as means $+/$-SEM for $\mathrm{n}=4$ and analysed by oneway ANOVA where $* * \mathrm{P}<0.01$ and $* * * \mathrm{P}<0.001$. (c) Cytokine proteins levels in human monocyte-derived macrophages pre-treated with various amounts of defactinib for $1 \mathrm{~h}$, followed by stimulation with LPS for $24 \mathrm{~h}$.

\section{Supplementary Figure 8. PYK2 inhibition in Hh/anti-IL10R-model of murine colitis.}

(a) Defactinib treatment regime during the initiation phase of mouse $\mathrm{Hh}+$ anti-IL-10R colitis. defactinib. (c) PYK2 autophosphorylation (pY402) in vehicle or defactinib treated mice (6 mice each) assessed by western blot analysis. Lysates from LPS stimulated GM-BMDMs 
included as a positive control. (d) Gene expression levels in colon tissue, leukocytes, monocytes/macrophages from vehicle or defactinib Hh/anti-IL10R treated mice. (e) Gating strategy for (b). Data in (b) and (d) are shown as means + -SEM. $* P<0.05$ and $* * P<0.01$ by unpaired Student t test.

\section{Supplementary Figure 9. PYK2 inhibition in UC biopsies.}

(a) Defactinib treatment of human biopsies from inflamed and non-inflamed sites of patients with ulcerative colitis. (b) Cell viability by TUNEL assay was measured in colon biopsies after $24 \mathrm{hr}$ treatment with defactinib.

Supplementary Figure 10. Proposed model of IRF5 activation by PYK2 in macrophages. genes. Serine kinases IRAK4, TAK1, and IKK $\beta$ have been proposed to phosphorylate and activate IRF5 downstream of the TLR-MyD88 pathway $^{12-15}$, while IKK $\alpha$ and Lyn negatively regulated IRF5 ${ }^{19,62}$. Dectin-1 stimulation by whole glucan particles also leads to IRF5 mediated transcription and is likely to be Syk-dependent. 


\section{References}

799 1. Jostins, L. et al. Host-microbe interactions have shaped the genetic architecture of $800 \quad$ inflammatory bowel disease. Nature 491, 119-24 (2012).

801 2. Huang, H. et al. Fine-mapping inflammatory bowel disease loci to single-variant $802 \quad$ resolution. Nature 547, 173-178 (2017).

803 3. Smillie, C. S. et al. Intra- and Inter-cellular Rewiring of the Human Colon during Ulcerative Colitis. Cell 178, 714-730.e22 (2019).

4. Krausgruber, T. et al. IRF5 promotes inflammatory macrophage polarization and TH1TH17 responses. Nat. Immunol. 12, 231-238 (2011).

807 5. Takaoka, A. et al. Integral role of IRF-5 in the gene induction programme activated by Toll-like receptors. Nature 434, 243-9 (2005).

809 6. Yang, L., Feng, D., Bi, X., Stone, R. C. \& Barnes, B. J. Monocytes from Irf5-/- mice have an intrinsic defect in their response to pristane-induced lupus. J. Immunol. 189, $3741-50$ (2012).

812 7. GTEx Consortium et al. Genetic effects on gene expression across human tissues. Nature 550, 204-213 (2017).

814 8. Pandey, S. P., Yan, J., Turner, J. R. \& Abraham, C. Reducing IRF5 expression attenuates colitis in mice, but impairs the clearance of intestinal pathogens. Mucosal Immunol. 12, 874-887 (2019).

817 9. Corbin, A. L. et al. IRF5 promotes intestinal inflammation by guiding monocyte 818 differentiation towards a pathogenic CD11c+ macrophage phenotype. bioRxiv 601963 $819 \quad$ (2019) doi:10.1101/601963.

820 10. Ryzhakov, G., Eames, H. L. \& Udalova, I. a. Activation and Function of Interferon Regulatory Factor 5. J. Interf. Cytokine Res. 35, 71-78 (2015). 
11. Chang Foreman, H.-C., Van Scoy, S., Cheng, T.-F. \& Reich, N. C. Activation of interferon regulatory factor 5 by site specific phosphorylation. PLoS One 7, e33098 (2012).

12. Cushing, L. et al. IRAK4 kinase activity controls Toll-like receptor-induced inflammation through the transcription factor IRF5 in primary human monocytes. $J$. Biol. Chem. 292, 18689-18698 (2017).

13. Bergstrom, B. et al. TLR8 Senses Staphylococcus aureus RNA in Human Primary Monocytes and Macrophages and Induces IFN- Production via a TAK1-IKK -IRF5 Signaling Pathway. J. Immunol. 195, doi:10.4049/jimmunol.1403176 (2015).

14. Lopez-Pelaez, M. et al. Protein kinase IKK $\beta$-catalyzed phosphorylation of IRF5 at Ser462 induces its dimerization and nuclear translocation in myeloid cells. Proc. Natl. Acad. Sci. U. S. A. 111, 17432-7 (2014).

15. Ren, J., Chen, X. \& Chen, Z. J. IKK $\beta$ is an IRF5 kinase that instigates inflammation. Proc. Natl. Acad. Sci. U. S. A. 111, 17438-43 (2014).

16. Lin, R., Yang, L., Arguello, M., Penafuerte, C. \& Hiscott, J. A CRM1-dependent nuclear export pathway is involved in the regulation of IRF-5 subcellular localization. J. Biol. Chem. 280, 3088-95 (2005).

17. Zhao, Y. et al. Microbial recognition by GEF-H1 controls IKK $\varepsilon$ mediated activation of IRF5. Nat. Commun. 10, 1349 (2019).

18. Balkhi, M. Y., Fitzgerald, K. A. \& Pitha, P. M. IKK $\alpha$ negatively regulates IRF-5 function in a MyD88-TRAF6 pathway. Cell. Signal. 22, 117-127 (2010).

19. Ban, T. et al. Lyn Kinase Suppresses the Transcriptional Activity of IRF5 in the TLRMyD88 Pathway to Restrain the Development of Autoimmunity. Immunity (2016) doi:10.1016/j.immuni.2016.07.015.

20. Okigaki, M. et al. Pyk2 regulates multiple signaling events crucial for macrophage 
morphology and migration. Proc. Natl. Acad. Sci. U. S. A. 100, 10740-5 (2003).

21. Krausgruber, T. et al. IRF5 is required for late-phase TNF secretion by human dendritic cells. Blood 115, 4421-4430 (2010).

22. Saliba, D. G. et al. IRF5:RelA interaction targets inflammatory genes in macrophages. Cell Rep. 8, 1308-17 (2014).

23. Dranchak, P. et al. Profile of the GSK published protein kinase inhibitor set across ATP-dependent and-independent luciferases: implications for reporter-gene assays. PLoS One 8, e57888 (2013).

24. Cheng, T.-F. et al. Differential Activation of IFN Regulatory Factor (IRF)-3 and IRF-5 Transcription Factors during Viral Infection. J. Immunol. 176, (2006).

25. Pandey, A. K. et al. NOD2, RIP2 and IRF5 Play a Critical Role in the Type I (2009).

26. Liu, J. Z. et al. Association analyses identify 38 susceptibility loci for inflammatory bowel disease and highlight shared genetic risk across populations. Nat. Genet. 47,

27. Fairfax, B. P. et al. Innate immune activity conditions the effect of regulatory variants upon monocyte gene expression. Science 343, 1246949 (2014). and regulates MyD88-mediated NF-kappaB activation in macrophages. J. Leukoc. Biol. 87, 415-23 (2010).

29. AR, A., M, C., EF, T. \& RK, G. The tyrosine kinase Pyk2 mediates 180, 5636-5644 (2008).

871 30. Wang, G. G. et al. Quantitative production of macrophages or neutrophils ex vivo 
using conditional Hoxb8. Nat. Methods 2006 34 3, 287-293 (2006).

31. Massimino, M. et al. IRF5 is a target of BCR-ABL kinase activity and reduces CML cell proliferation. Carcinogenesis 35, 1132-1143 (2014).

32. Lipinski, C. A. \& Loftus, J. C. Targeting Pyk2 for therapeutic intervention. Expert Opin. Ther. Targets 14, 95-108 (2010).

33. Müller, S. et al. Donated chemical probes for open science. Elife 7, (2018).

34. Lin, H.-M. et al. Effect of FAK inhibitor VS-6063 (defactinib) on docetaxel efficacy in prostate cancer. Prostate (2018) doi:10.1002/pros.23476.

35. Patel, M. R. et al. Abstract A69: Phase 1/1b study of the FAK inhibitor defactinib (VS6063 ) in combination with weekly paclitaxel for advanced ovarian cancer. Mol.

Cancer Ther. 12, A69-A69 (2013).

36. Slack-Davis, J. K. et al. Cellular Characterization of a Novel Focal Adhesion Kinase Inhibitor. J. Biol. Chem. 282, 14845-14852 (2007).

37. Kuprash, D. V et al. Similarities and differences between human and murine TNF promoters in their response to lipopolysaccharide. J. Immunol. 162, 4045-52 (1999).

38. del Fresno, C. et al. Interferon- $\beta$ production via Dectin-1-Syk-IRF5 signaling in dendritic cells is crucial for immunity to C. albicans. Immunity 38, 1176-86 (2013).

39. Kullberg, M. C. et al. IL-23 plays a key role in Helicobacter hepaticus-induced T celldependent colitis. J. Exp. Med. 203, 2485-94 (2006).

40. Weiss, M. et al. IRF5 controls both acute and chronic inflammation. Proc. Natl. Acad. Sci. 112, 11001-11006 (2015).

41. West, N. R. et al. Oncostatin M drives intestinal inflammation and predicts response to tumor necrosis factor-neutralizing therapy in patients with inflammatory bowel disease. Nat. Med. 23, 579-589 (2017).

896 42. Zhao, M., Finlay, D., Zharkikh, I. \& Vuori, K. Novel Role of Src in Priming Pyk2 
Phosphorylation. PLoS One 11, e0149231 (2016).

43. Wu, S. S., Jácamo, R. O., Vong, S. K. \& Rozengurt, E. Differential regulation of Pyk2 phosphorylation at Tyr-402 and Tyr-580 in intestinal epithelial cells: roles of calcium, Src, Rho kinase, and the cytoskeleton. Cell. Signal. 18, 1932-40 (2006).

901

44. Kelly, E. K., Wang, L. \& Ivashkiv, L. B. Calcium-activated pathways and oxidative burst mediate zymosan-induced signaling and IL-10 production in human macrophages. J. Immunol. 184, 5545-52 (2010).

904

45. Barnes, B. J., Kellum, M. J., Field, A. E. \& Pitha, P. M. Multiple regulatory domains of IRF-5 control activation, cellular localization, and induction of chemokines that mediate recruitment of T lymphocytes. Mol. Cell. Biol. 22, 5721-40 (2002).

46. Chen, W. et al. Insights into interferon regulatory factor activation from the crystal structure of dimeric IRF5. Nat. Struct. Mol. Biol. 15, 1213-20 (2008).

47. Shimizu, T. et al. A first-in-Asian phase 1 study to evaluate safety, pharmacokinetics and clinical activity of VS-6063, a focal adhesion kinase (FAK) inhibitor in Japanese patients with advanced solid tumors. Cancer Chemother. Pharmacol. 77, 997-1003 (2016).

48. Duffau, P. et al. Promotion of Inflammatory Arthritis by Interferon Regulatory Factor 5 in a Mouse Model. Arthritis Rheumatol. (Hoboken, N.J.) 67, 3146-57 (2015).

49. Seneviratne, A. N. et al. Interferon Regulatory Factor 5 Controls Necrotic Core Formation in Atherosclerotic Lesions by Impairing Efferocytosis. Circulation 136, 1140-1154 (2017).

50. Liao, M. et al. The landscape of lung bronchoalveolar immune cells in COVID-19 revealed by single-cell RNA sequencing. medRxiv 2020.02.23.20026690 (2020) doi:10.1101/2020.02.23.20026690.

921 51. Dobin, A. et al. STAR: ultrafast universal RNA-seq aligner. Bioinformatics 29, 15-21 
(2013).

923

924

925

926

927

928

929

930

931

932

933

934

935

936

937

938

939

940

941

942

943

944

945

946

52. Liao, Y., Smyth, G. K. \& Shi, W. featureCounts: an efficient general purpose program for assigning sequence reads to genomic features. Bioinformatics 30, 923-930 (2014).

53. Love, M. I., Huber, W. \& Anders, S. Moderated estimation of fold change and dispersion for RNA-seq data with DESeq2. Genome Biol. 15, 550 (2014).

54. Ryzhakov, G. et al. Cross-species analysis reveals evolving and conserved features of the nuclear factor $\kappa \mathrm{B}(\mathrm{NF}-\kappa \mathrm{B})$ proteins. J. Biol. Chem. 288, 11546-54 (2013).

55. Carlson, S. M. \& White, F. M. Labeling and identification of direct kinase substrates. Sci. Signal. 5, pl3 (2012).

56. Fischer, R. \& Kessler, B. M. Gel-aided sample preparation (GASP)--a simplified method for gel-assisted proteomic sample generation from protein extracts and intact cells. Proteomics 15, 1224-9 (2015).

57. Wessel, D. \& Flügge, U. I. A method for the quantitative recovery of protein in dilute solution in the presence of detergents and lipids. Anal. Biochem. 138, 141-3 (1984).

58. Montoya, A., Beltran, L., Casado, P., Rodríguez-Prados, J.-C. \& Cutillas, P. R. Characterization of $\mathrm{TiO}_{2}$ enrichment method for label-free quantitative phosphoproteomics. Methods 54, 370-8 (2011).

59. Davis, S. et al. Expanding Proteome Coverage with CHarge Ordered Parallel Ion aNalysis (CHOPIN) Combined with Broad Specificity Proteolysis. J. Proteome Res. 16, 1288-1299 (2017).

60. Vizcaíno, J. A. et al. 2016 update of the PRIDE database and its related tools. Nucleic Acids Res. 44, D447-D456 (2016).

61. Izcue, A. et al. Interleukin-23 restrains regulatory T cell activity to drive T celldependent colitis. Immunity 28, 559-70 (2008).

62. Balkhi, M. Y., Fitzgerald, K. A. \& Pitha, P. M. IKK $\alpha$ negatively regulates IRF-5 
bioRxiv preprint doi: https://doi.org/10.1101/2020.05.24.113076; this version posted May 27, 2020. The copyright holder for this preprint

(which was not certified by peer review) is the author/funder. All rights reserved. No reuse allowed without permission.

function in a MyD88-TRAF6 pathway. Cell. Signal. 22, 117-127 (2010).

948 
Fiqurebiqxiv preprint doi: https://doi.org/10.1101/2020.05.24.113076; this version posted May 27, 2020. The copyright holder for this preprint (which was not certified by peer review) is the author/funder. All rights reserved. No reuse allowed without permission.

a

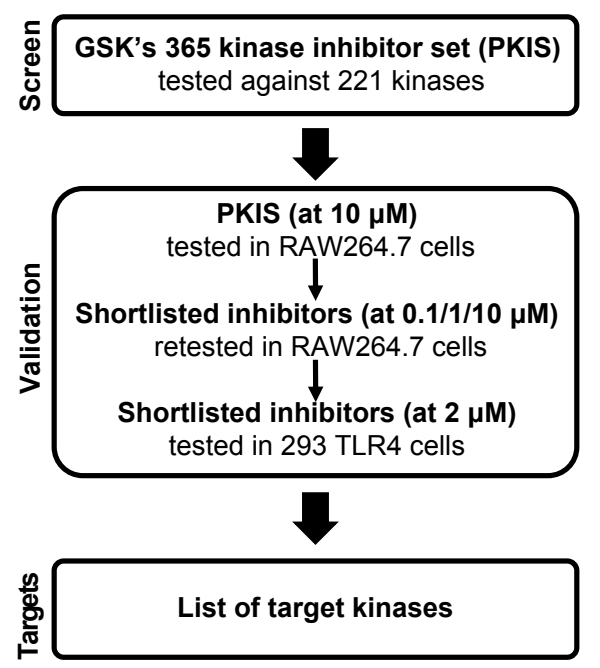

Target kinases

PDK1 HIPK4

PKC- $\alpha$ JNK2

PRKD1 IKK- $\varepsilon$

PRKD2 MST1

PRKD3 MST2

ARK5 FYN

MARK1 HCK

MARK3 IGF1R

MARK4 INSR

TBK1 PYK2

CK1 $\alpha$ ROS

CLK2 SRC

DYRK1A TNK1

DYRK1B TRKA

GSK3 $\alpha$ TRKB

GSK3 $\beta$ TRKC

HIPK1 IRAK4

C

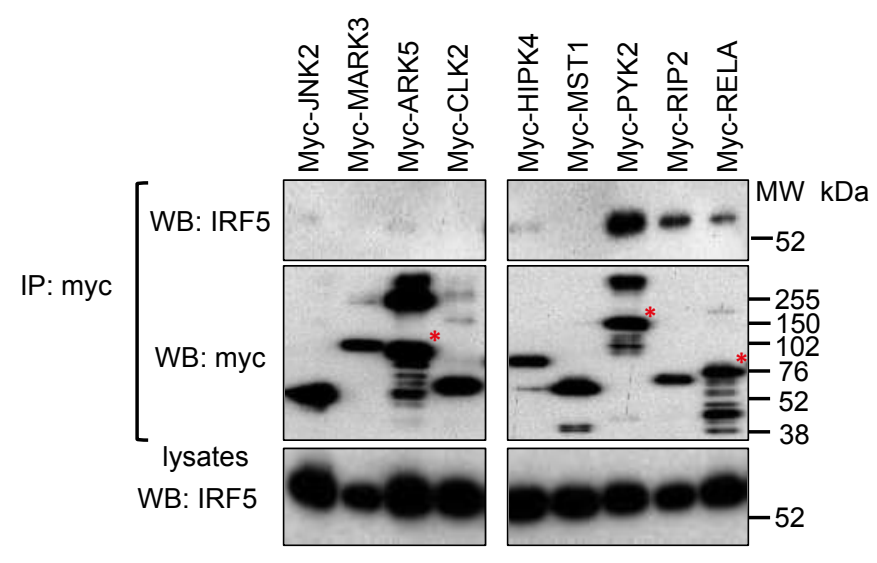

b

TNF-luc

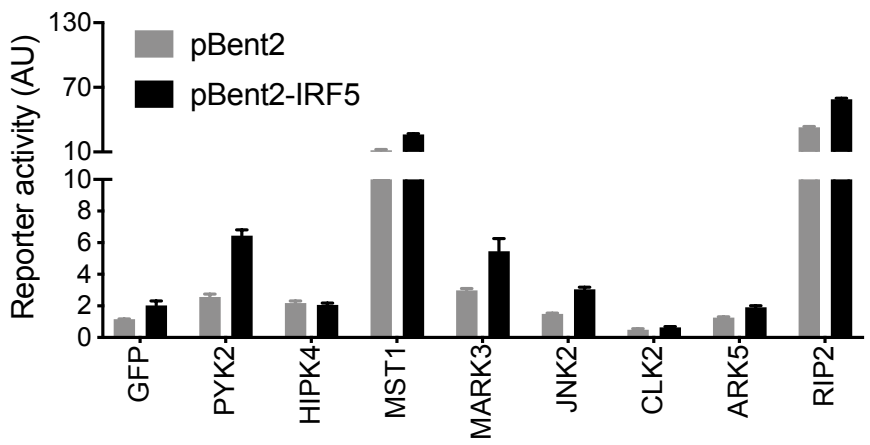

d

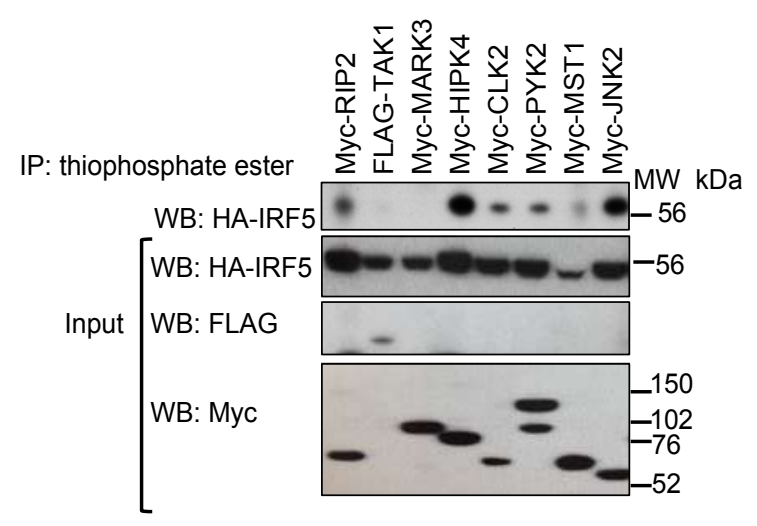


biogxiv preprint doi: https://doi.org/10.1101/2020.05.24.113076; this version posted May 27, 2020. The copyright holder for this preprint

Figure 2 (which was not certified by peer review) is the author/funder. All rights reserved. No reuse allowed without permission.

a

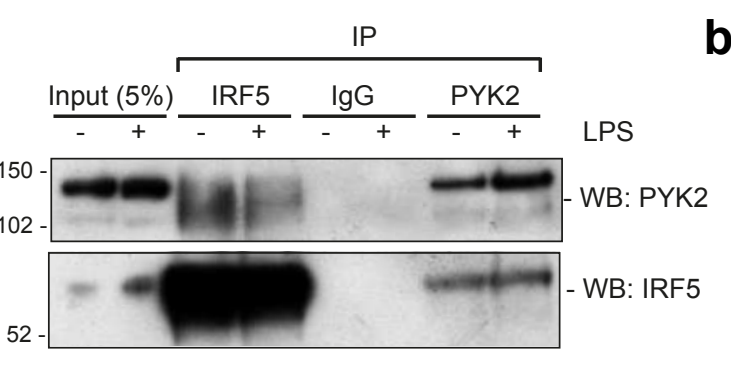

b

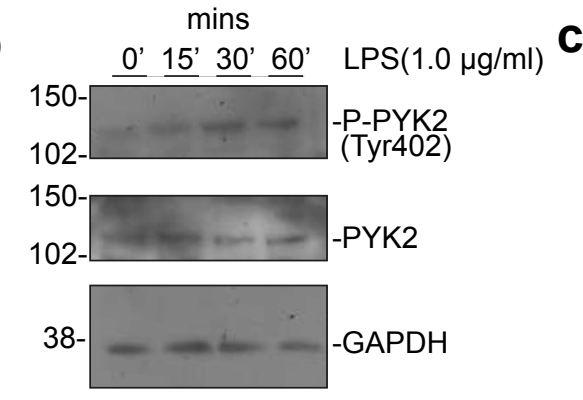

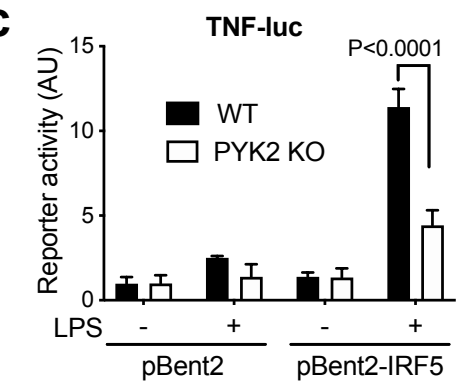

d IRF5

pol II

control (lgG)

e

116
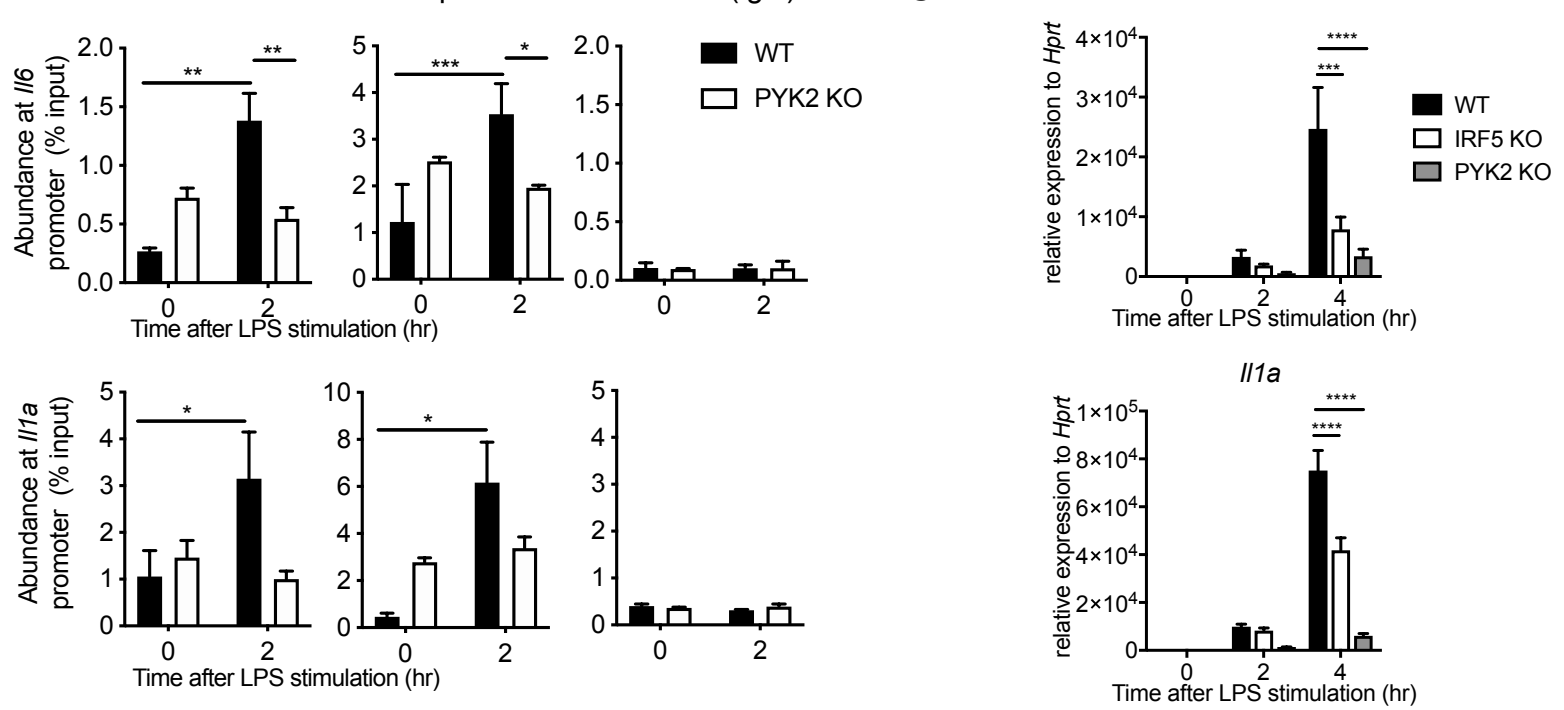

f

\begin{tabular}{|c|c|}
\hline WT RAW264.7 & PYK2 KO RAW264.7 \\
\hline- & S56 \\
\hline Y171 & - \\
\hline S172 & S172 \\
\hline S300 & S300 \\
\hline- & Y312 \\
\hline Y334 & Y334 \\
\hline S445 & S445 \\
\hline
\end{tabular}

g

pY171

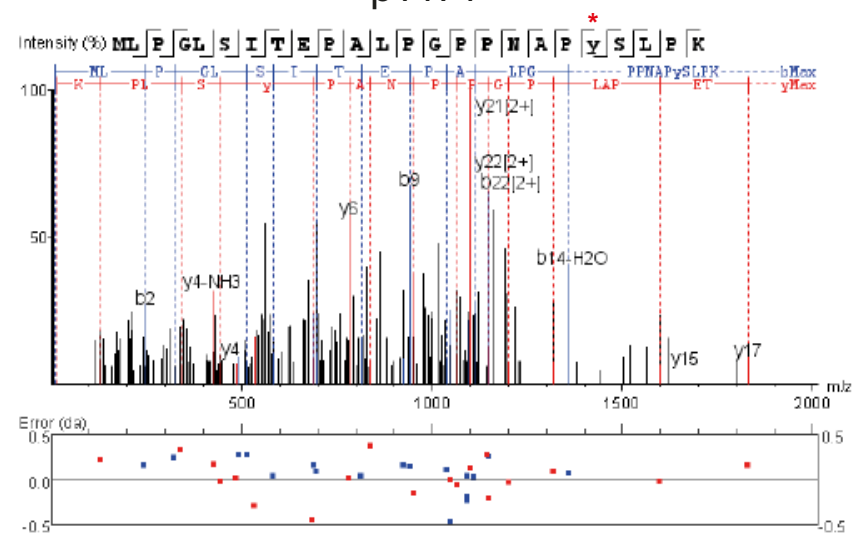

$\mathbf{h}$ TNF-luc (HEK-TLR4)

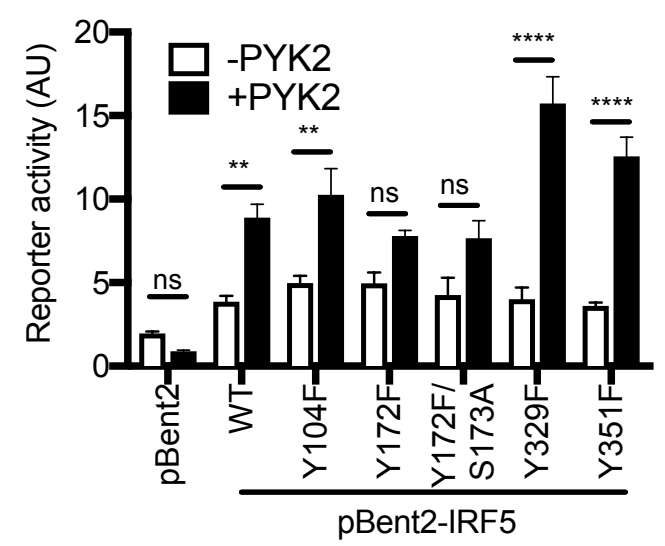

i

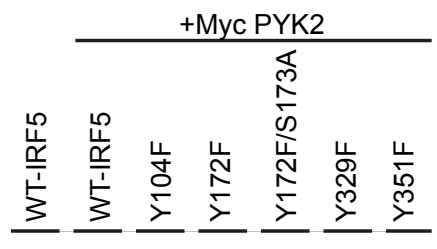

IP:thiophoshate ester

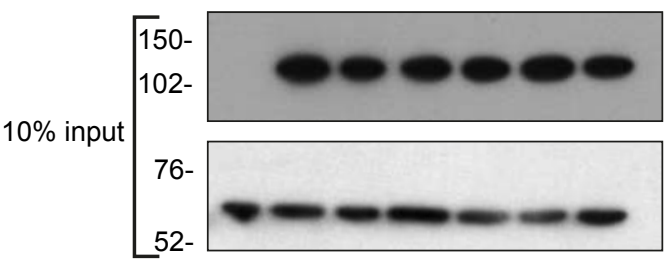

-WB: Flag-IRF5 
Figurebi32xiv preprint doi: https://doi.org/10.1101/2020.05.24.113076; this version posted May 27, 2020. The copyright holder for this preprint a (which was not certified by peer review) is the author/funder. All rights reserved. No reuse allowed without permission.
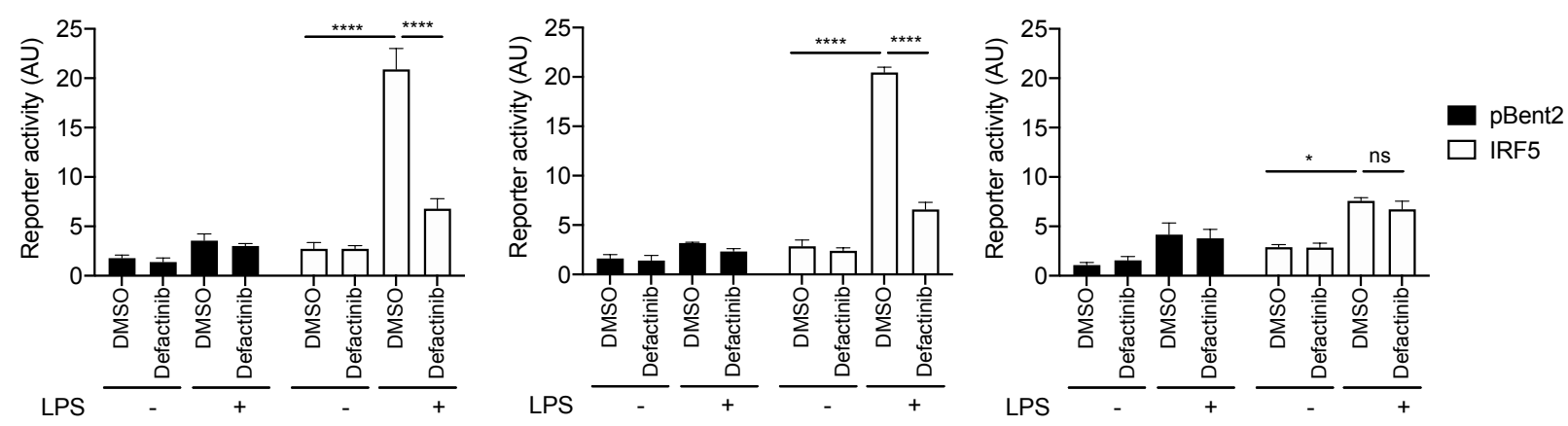

b

C

IRF5

pol II

IRF5

pol II
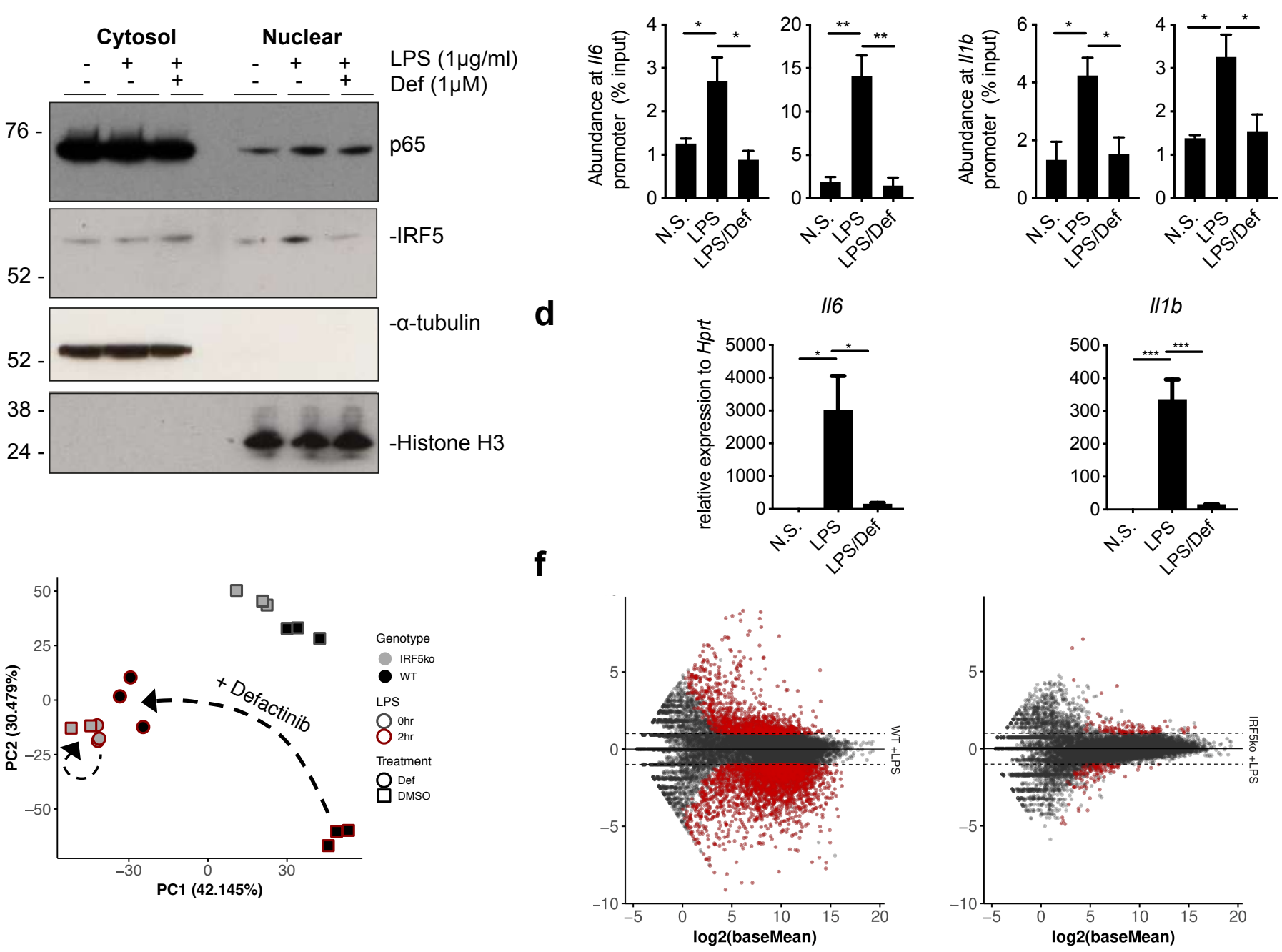

$\| 1 b$

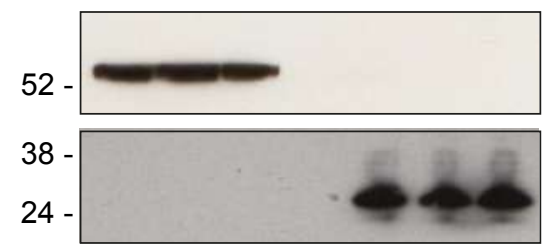

-a-tubulin

-Histone H3

e

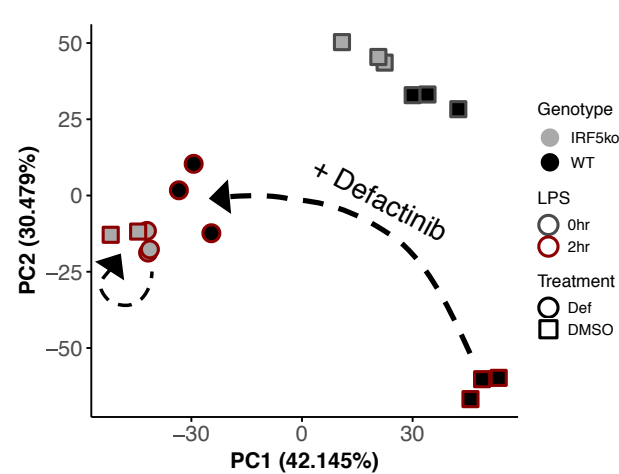

g

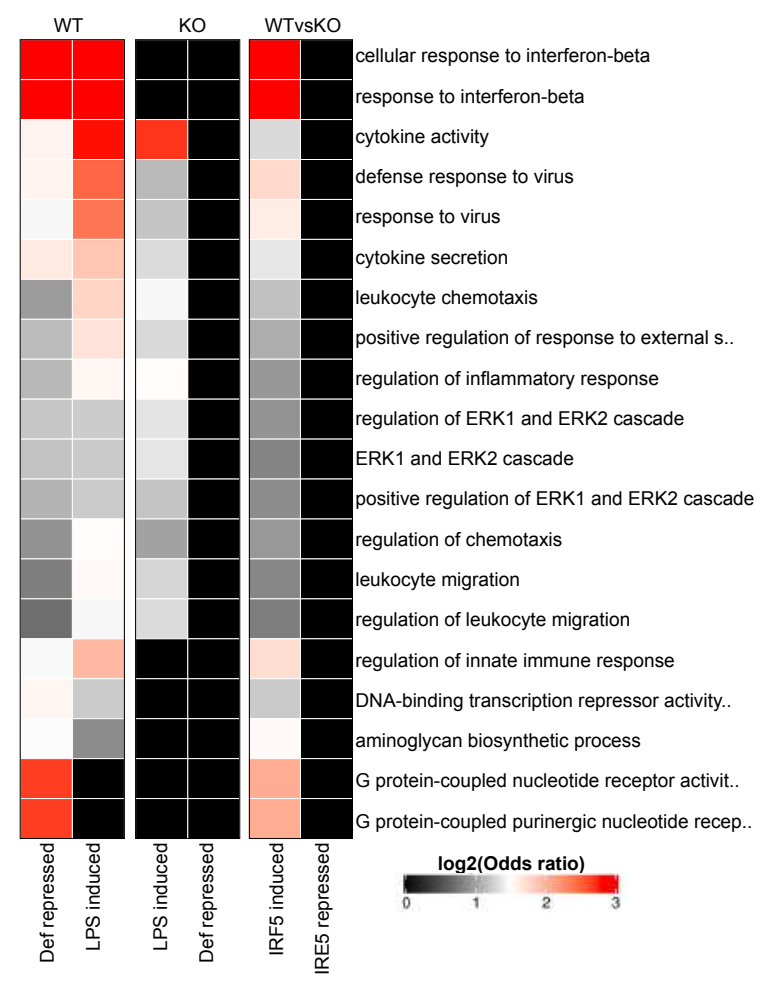

h

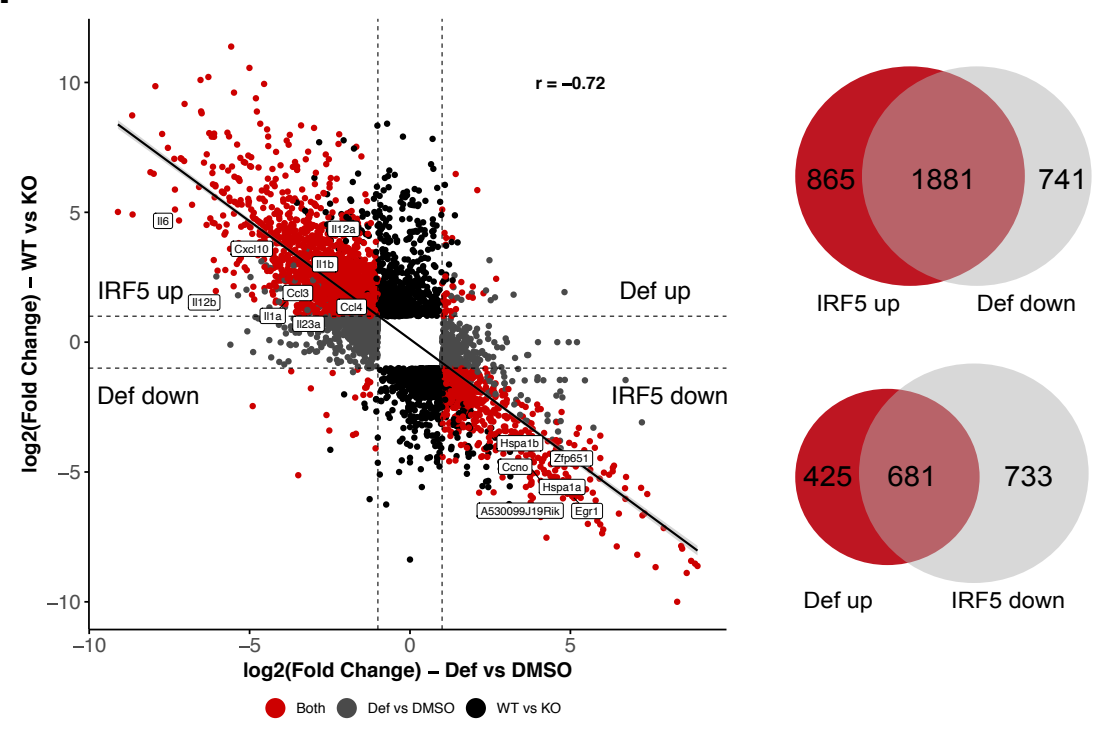


Fiqure 4poRiv preprint doi: https://doi.org/10.1101/2020.05.24.113076; this version posted May 27, 2020. The copyright holder for this preprint

a

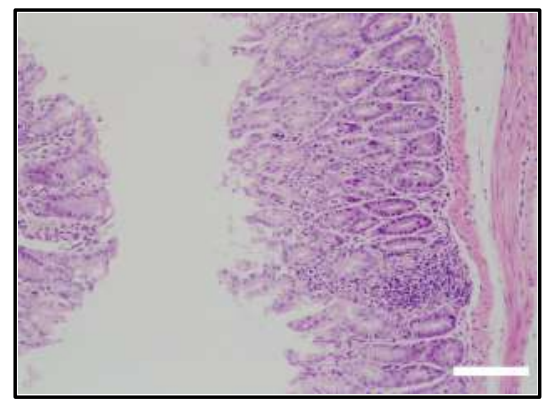

Vehicle (which was not certified by peer review) is the author/funder. All rights reserved. No reuse allowed without permission.

b

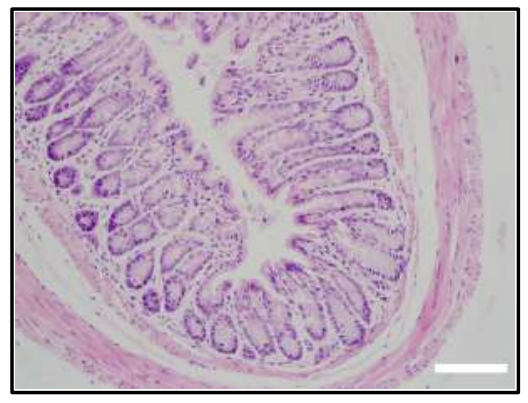

Defactinib

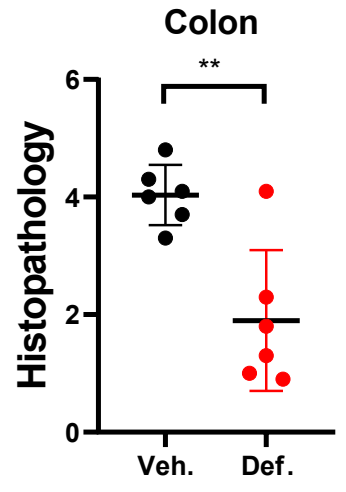

C

Colon LPL \#

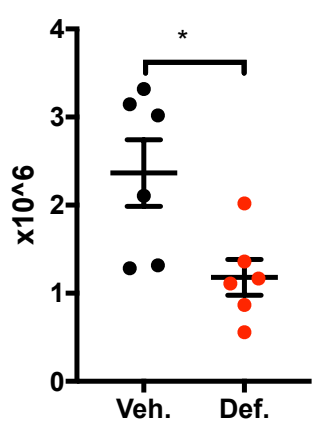

d
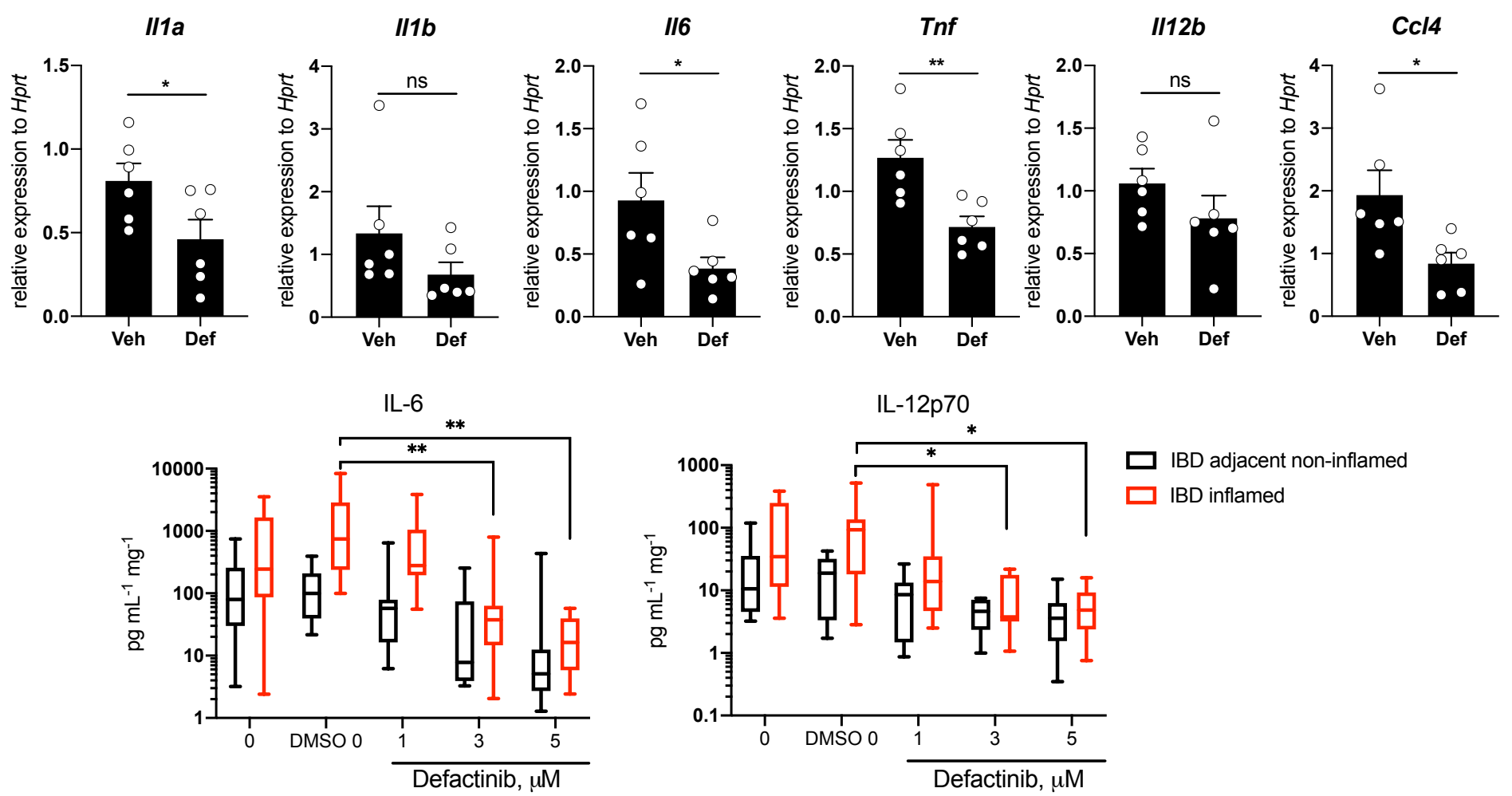

$\square$ IBD adjacent non-inflamed

IL-1b

IL-10
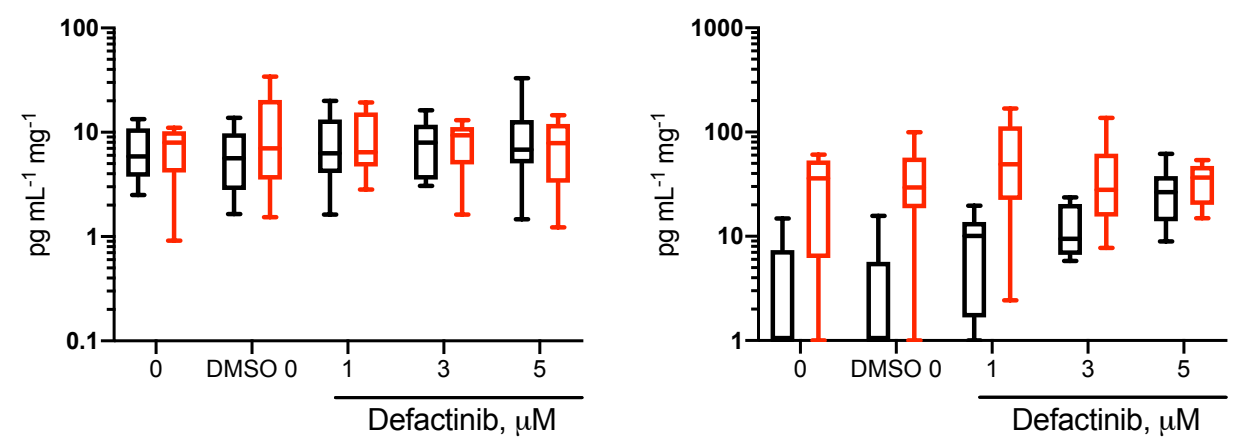